\title{
INITIATION OF SYNAPSIS AND INTERLOCKING OF CHROMOSOMES DURING ZYGOTENE IN BOMBYX SPERMATOCYTES
}

by

\author{
SØREN WILKEN RASMUSSEN
}

Department of Physiology, Carlsberg Laboratory

Gamle Carlsberg Vej 10, DK-2500 Copenhagen Valby

Keywords: Meiosis, synaptonemal complex

\begin{abstract}
Initiation of synapsis and interlocking of chromosomes have been analysed on spread and silver stained chromosome complements from Bombyx spermatocytes. The material comprises 270 zygotene and 125 pachytene nuclei. Two types of associations preceding synaptonemal complex formation were detected: The first type involved short subterminal segments of homologous chromosomes while the second occurred between the telomeric attachment plaques of homologous as well as nonhomologous chromosomes. The possible role of the two types of initial associations in synaptonemal complex formation is discussed.

The occurrence and resolution of chromosome interlocking have been monitored during the entire zygotene stage. Interlockings form and resolve throughout zygotene. The estimated mean number of interlockings is 4.2 per nucleus at zygotene while only 3 presumptive interlockings were found in 125 pachytene nuclei. In individual zygotene nuclei up to $61 \%$ of the chromosomes are affected by interlocking. The mechanism for resolution of interlockings is discussed
\end{abstract}

\section{INTRODUCTION}

It is well documented in a variety of organisms that synapsis and synaptonemal complex (SC) formation during the zygotene stage of meiotic prophase yield an apparently precise alignment of homologous chromosomes at pachytene (54). In heterozygotes for chromosomal rearrangements, synapsis of the rearranged chromosome segments and their homologous undisturbed segments usually leads to the expected inversion loop, duplication buckle, translocation quadrivalent etc. confirming the specificity of synapsis at zygotene. It has further been demonstrated that the pairing pattern achieved at zygotene is not irreversible but is subject to subsequent synaptic adjustment $(39,40,53)$ of heteromorphous bivalents in rearrangement het- erozygotes and conversion of multivalent chromosome associations into bivalents in polyploid species $(43,45)$. In both cases the secondary SC formation appears to be indifferent to homology $(39,40,43,53)$.

Information on the appearance and behaviour of paired chromosomes at pachytene is available for a large number of organisms (54) and constitutes the main body of experimental evidence for the precision of synapsis at zygotene. Much less is known about the initiation of synapsis at the beginning of zygotene. It is a common observation that $\mathrm{SC}$ formation during zygotene in diploids occurs only between homologous chromosomes. It is, however, not experimentally verified whether this is because SC formation per se requires homology of the

Abbreviations: IL = interlocking; $\mathrm{LC}=$ lateral component; $\mathrm{SC}=$ synaptonemal complex. 
pairing chromosome segments, or because previous specific associations have been established at the chromatin level holding homologous chromosomes in register prior to SC formation, as originally proposed by COMINGS and RIGGS (8) and recently by HASENKAMPF (21) and Weith and Traut (53).

The few studies of synaptic initiation at zygotene $(21,22,35,37)$ seem to support the notion that specific recognition of homologues is not directly coupled to the actual formation of the SC. As early as 1969 MOENS (36) showed that SC formation in the grasshopper, Locusta migratoria, is initiated a short distance from the attached telomeres and that the first visible contact between homologues occurred when they had approached to a distance of about 300 nm, i.e., 2-3 times the width of the completed SC. This observation has recently been corroborated by the work of HASENKAMPF $(21,22)$ and LOIDL and JONES (35) on synapsis and synaptic initiation in the long chromosomes of Tradescantia and Allium.

A second aspect of chromosome pairing which has received attention in recent studies is the frequent occurrence of interlocked chromosomes at zygotene and the absence of such entanglements at pachytene. The key problem has been precisely formulated by CALLAN and PERRY (7): “ It is easy enough to account for interlocking; the real puzzle is how interlocking is normally avoided by chromosomes which start their synapsis at both telomeres".

When synapsis of two homologous chromosomes is initiated at two different sites, other chromosomes located between their unpaired regions will be trapped and an interlocking (IL) is formed. If left uncorrected at zygotene, the presence of an IL prevents completion of chromosome pairing and SC formation and thus reduces the probability of crossing over in the homologues involved in the IL. At diplotene some ILs will resolve upon disassembly of the SC, while others may persist until metaphase I due to crossing over and chiasma formation on both sides of the unpaired regions enclosing other chromosomes. In a study of the behaviour of interlocked bivalents at metaphase and anaphase I, Buss and HENDERSON (5) showed that stable bipolar orientation was achieved by most interlocked bivalents and that nondisjunction occurred only in few cells. This analysis was, however, performed on the acrocentric bivalents of Locusta migratoria $(2 n=22+X$; mean number of chiasmata per cell $=14)(50)$ and may not be representative of the situation in organisms with higher chiasma frequencies and different location of the centromeres. It is more likely that the presence of interlocked bivalents at metaphase I may cause segregational disorder by impeding proper bipolar orientation of homologous kinetochores especially when several chromosomes and bivalents are involved in a multiple IL.

The fact that ILs are reported to be extremely rare at metaphase $I(5,6,31)$ is in striking contrast to the behaviour of chromosomes during synapsis. In the organisms so far studied, the homologous chromosomes are located at random in the nucleus prior to synapsis (54) and are brought together by undirected, random movements. Important in this process is the binding of the telomeres to the nuclear envelope and the subsequent congression of the attached telomeres giving rise to the so-called chromosome bouquet, typical of the zygotene stage of most organisms. Although this arrangement facilitates synapsis by bringing all telomeres and thus also homologous telomeres into proximity, it also increases the risk of entanglement in the form of interlocking. As interlocking of chromosomes occurs as the result of the random location of homologues and their subsequent undirected movements, considerable variation in the frequency of ILs is expected (and found) among different nuclei of the same organism.

The discrepancy between the rare occurrence of ILs at metaphase I and the high probability with which IL is expected to form during synapsis was explained when complete reconstructions of chromosome complements at zygotene became available $(28,29,42,44)$. The first reconstruction study demonstrating ILs at zygotene was carried out on the female silkworm, Bombyx mori, where three of the four reconstructed late zygotene nuclei contained one or more ILs. ILs were not detected in pachytene nuclei and it was concluded that ILs were either rare or were normally resolved prior to pachytene (42). More extensive reconstruc- 
tion analyses of human (44) and Bombyx (28) spermatocytes at late zygotene confirmed that chromosomes frequently interlock during synapsis and further showed that the interlocked chromosomes are released by breakage and rejoining of one or more of the involved chromosomes. Subsequently, ILs were found in reconstructed zygotene nuclei from human oocytes (3), Secale (1), Triticum $(24,30)$, Sordaria (58) and Coprinus (29) among others. In all cases, ILs were absent or present at very low frequencies in pachytene nuclei, confirming the initial observations in Bombyx and Homo and adding evidence to the conception that the formation and resolution of ILs are normal events during synapsis.

The number of nuclei which has been examined by serial sectioning and three dimensional reconstruction is small, the studies referred to above being based on 1 to 10 nuclei, mostly at late zygotene. In addition, problems are often encountered when attempts are made to reconstruct unpaired lateral components (LC) of the SC at early and mid zygotene. Some of these limitations were overcome by the introduction and refinement of a method by which the entire SC and LC complement of a nucleus can be analysed in a two dimensional spread after contrasting with ammoniacal silver or phosphotungstic acid. The spreading methods have proven very successful for pachytene complements (54) whereas the interpretation of spread zygotene complements has been difficult. The lack of a third dimension in the spreads, introduces ambiguity in the analysis of interlocked and entangled chromosomes and may explain the scarcity of information available from spread zxgotene nuclei. Except for a recent paper on synapsis in wheat (27), the analyses have been based on numbers of nuclei comparable to those investigated by three-dimensional reconstruction.

Interlocked chromosomes in spread complements have been observed in Secale (16), Zea $(14,15)$, Triticum tri-isosomic for the long arm of chromosome 5BL (55), Bombyx (4), Mus (9, $11,20)$ and possibly in Chinese hamster (10). Published micrographs of spread zygotene complements of Tradescantia $(21,22)$ indicate the occurrence of ILs and extensive entanglement of the pairing chromosomes. An extensive study of synapsis in hexaploid wheat (27) finally revealed a large number of ILs in all analysed mid zygotene nuclei. A single zygotene nucleus examined in detail for the presence of ILs contained 5 chromosome ILs, 15 bivalent ILs, as well as a number of configurations indicating the presence of additional ILs. The fact that most detailed observations on zygotene complements have been carried out on plant chromosomes may in part be due to the superior stainability of unpaired LCs in plants compared to animals.

A favorable situation for a quantitative analysis of synapsis exists in the male silkworm $(2 n=$ 56 ) in which cells in a particular stage of meiosis can be obtained in large quantities. The rather short chromosomes (range $4.2-10.4 \mu \mathrm{m}, 28$ ) reduce the risk of chromosome breakage during spreading and offer a potentially simple system for analysis. Finally, a detailed analysis from three dimensional reconstructions is available (28) comprising a reference for the observations made on the spread chromosomes.

The present study, which is based on 270 fully traced zygotene and 125 pachytene complements, concentrates on the progression of synapsis from leptotene to pachytene with special emphasis on the initial contact between homologous chromosomes and how this relates to the subsequent formation of the SC. Second$l y$, the formation and resolution of ILs have been monitored during the entire zygotene stage in an attempt to precisely estimate the frequency and kinetics of these events.

\section{MATERIALS AND METHODS}

The silkworms (hybrids between strains Lyon 200 and 300 ) were kindly provided by Professor J.M. LEGAY, Laboratoire de Biométrie, Université Claude Bernard, France. Since 1982 the hybrids have been maintained as a random breeding population in the laboratory on an artificial diet (Silkmate E, Nihon Nosan Kogyo KK, Japan). Hatching of diapause eggs stored for up to 6 months at $5{ }^{\circ} \mathrm{C}$ was induced by incubation in $16 \% \mathrm{HCl}$ at $46^{\circ} \mathrm{C}$ for $6-7 \min (46)$. Hatching occurred 10-14 days later.

Spread complements were prepared from 4th instar male larvae in the following way: 
The testes were removed from the 5 th abdominal segment and placed in a depression slide in 4-5 drops of Dulbecco's modified Eagles medium (Flow Laboratories U.K.) containing 2 $\mathrm{mM}$-disodium-ethylenediamine-tetracetic-acid (EDTA) and $0.1 \%$ bovine serum albumine (BSA). The fat bodies were removed and the testes ruptured to release the 64-cell clusters of primary spermatocytes. As much of the dissection medium as possible was removed and 2-3 drops of spreading medium (30 mM-Na- $\mathrm{PO}_{4}$ buffer, 5 mM-EDTA, $0.025 \%$ Triton X-100 (Sigma), $0.03 \%$ Tex-I (dishwashing agent, Bluemøller AS, Odense, Denmark), pH 7.0) were added. After $0-60 \mathrm{sec}$ the partially lysed 64 -cell clusters were washed onto an Optilux coated slide (prepared as described in 27) by a flow of $6-10$ drops of fixative (4\% formaldehyde, $1.5 \%$ sucrose, $\mathrm{pH} \mathrm{8.2)} \mathrm{and} \mathrm{dried} \mathrm{at} 60^{\circ} \mathrm{C}$ for several hours. The slides were then carefully washed in distilled water for $2 \mathrm{~min}$, in $0.1 \%$ Kodak PhotoFlo 200 for $30 \mathrm{sec}$ and air dried in an upright position at room temperature. The final washing was found to be a critical step since the spread nuclei adhered poorly to the Optilux membrane and many nuclei were lost during the final rinse.

The spread chromosome complements were silver stained as described by BLOOM and GOODPASTURE (2). After staining, the Optilux membrane was transferred from the slide to a water surface, the entire area containing the spread nuclei was covered by single slot grids previously dipped in an $8 \%$ solution of "Klæb op" (a glue for mounting photographs) in heptane and the floating membrane picked/rolled up on a piece of Parafilm attached to the cylindrical surface of a small glass bottle.

The grids were inspected in the light microscope and those containing zygotene and pachytene complements selected for further analysis in the electron microscope. About 500 zygotene and 200 pachytene complements were photographed at a primary magnification of 1,500 times and printed to a final magnification of 6-7,000 times. About half of the zygotene and one third of the pachytene complements were rejected due to inadequate staining, excess or insufficient spreading, severe silver precipitation or apparent deformation/stretching of the chromosome complement. The length of LCs and SCs of the adequately preserved and stained complements were measured using a Hewlett Packard digitizer and calculator. The percentage of synapsis with the SC was then determined for

Table I. Mean lateral component (LC) length, its standard deviation and range for the spread zygotene and pachytene chromosome complements.

\begin{tabular}{lllll}
\hline $\begin{array}{l}\text { Percent } \\
\text { pairing }\end{array}$ & $\begin{array}{l}\text { Number of } \\
\text { nuclei }\end{array}$ & $\begin{array}{l}\text { Mean total } \\
\text { LC length } \\
(\mu \mathrm{m})\end{array}$ & $\begin{array}{l}\text { Standard } \\
\text { deviation } \\
(\mu \mathrm{m})\end{array}$ & Range \\
\hline $0-10$ & 6 & 386 & 67 & $346-506$ \\
$10-20$ & 10 & 446 & 85 & $361-643$ \\
$20-30$ & 17 & 426 & 61 & $305-527$ \\
$30-40$ & 16 & 395 & 54 & $319-489$ \\
$40-50$ & 15 & 487 & 102 & $347-680$ \\
$50-60$ & 28 & 415 & 57 & $317-591$ \\
$60-70$ & 33 & 455 & 82 & $346-619$ \\
$70-80$ & 39 & 442 & 88 & $313-698$ \\
$80-90$ & 40 & 447 & 74 & $361-661$ \\
$90-100$ & 66 & 437 & 69 & $334-642$ \\
\hline Pachytene 1 & 40 & 426 & 45 & $329-502$ \\
Pachytene 2 & 67 & 432 & 47 & $355-596$ \\
Pachytene 3 & 18 & 423 & 44 & $299-490$ \\
\hline
\end{tabular}




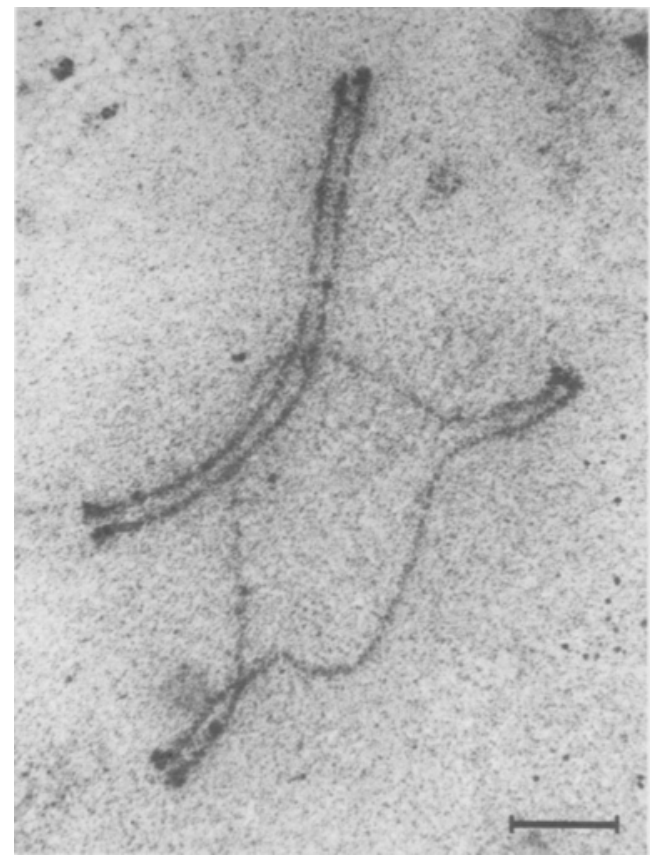

Figure 1. Bivalent interlocking. A fully paired bivalent is entrapped between the unpaired middle region of a bivalent. $(\mathrm{Bar}=1.0 \mu \mathrm{m})$

all zygotene complements and used to establish the developmental sequence as described in the following section.

\section{RESULTS}

\subsection{Interpretation of spread chromosome complements}

The arrangement of primary spermatocytes of Bombyx in clusters of 64 interconnected cells introduce some variation in the degree of spreading obtained after incubation in the spreading solution. The outer layer of cells in the cluster tends to be spread more than cells located in the center, yielding nuclei spread to varying extents. As can be seen from Table I, the range and standard deviation of the total LC length per nucleus are considerable. The pairing pattern of even extensively spread nuclei could, however, often be interpreted, and such nuclei were therefore included in the material. Discontinuities of LCs due to mechanical stretching were rare, although the electron density of the LCs after
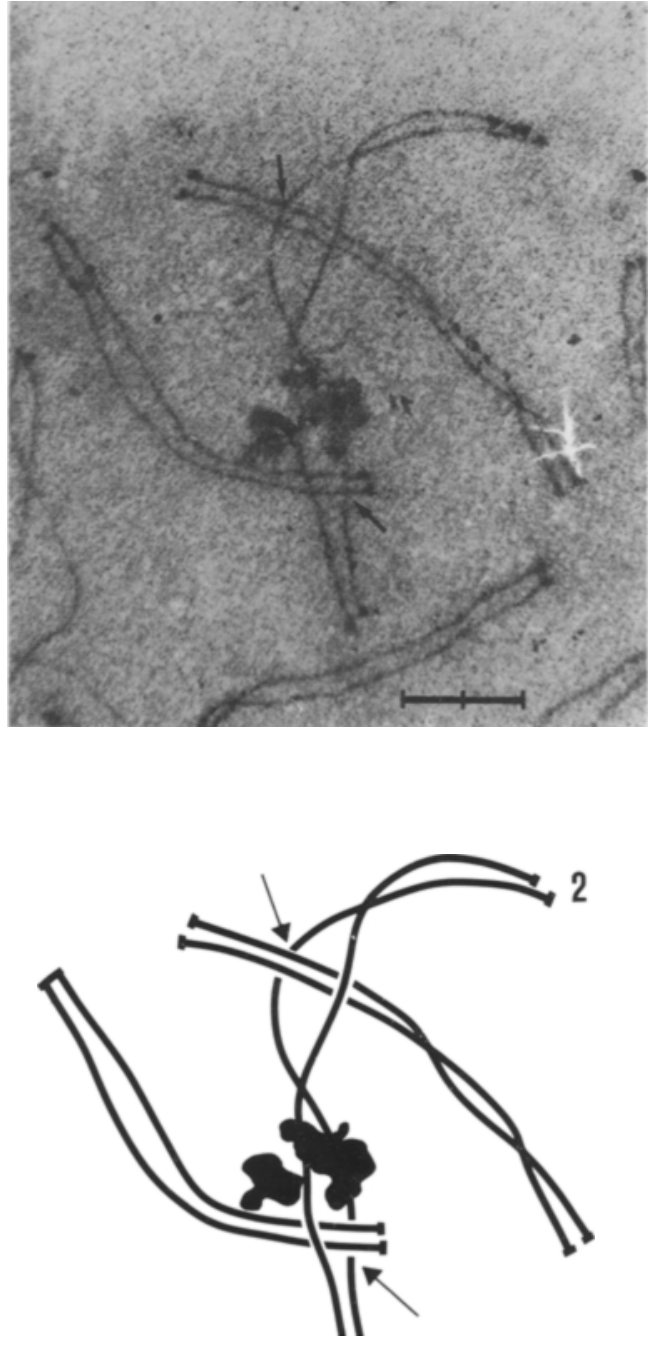

Figure 2. Double bivalent interlocking. A fully and a partially paired bivalent is entrapped between the LCs of bivalent 2 . The lower interlocking is clearly identifiable by the lack of staining in the LC segment of bivalent 2 which is covered by the SC of the interlocked bivalent (arrow). In the upper interlocking, the staining of the LC segment covered by the SC (arrow) is only slightly reduced. The interpretation of the topological relationship of the crossing LCs is shown in the drawing. $(\mathrm{Bar}=2.0 \mu \mathrm{m})$

silver staining appeared to be directly related to the extent of spreading, LCs in complements with smaller total length having a higher electron density than those of extensively spread 


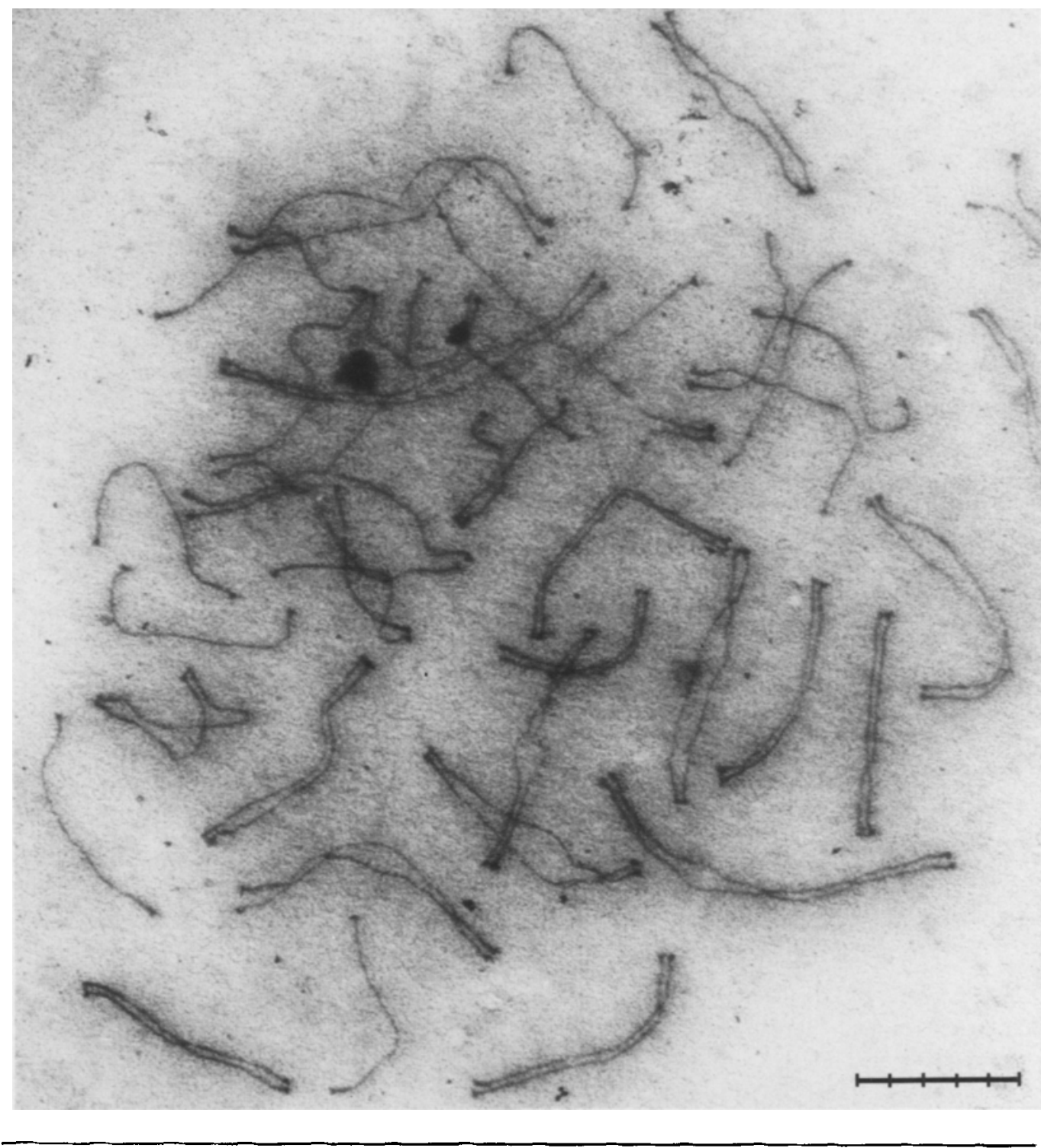

nuclei. In order to minimize the selection of nuclei, the only nuclei rejected were those which had suffered from the preparation or in which the chromosomes were entangled to an extent where the 56 chromosomes could not be identified by their LCs. The latter category may include nuclei which upon correction of the entanglement reach a normal pachytene morphology.

In analysing simple ILs as well as more complex entanglements, the topological relationship of overlapping SCs and unpaired LCs was resolvable in most cases by the difference in electron density of the crossing LCs (Figures 1 and 2), the upper $L C(s)$ being the more electron dense. The relationship of crossing unpaired LC segments was also often resolvable by differences in electron density alone. In both cases the interpretation was frequently aided by the abrupt bending of the interlocked LCs which is caused by the tension exerted on the interlocked LCs during spreading of the complement (Fig- 


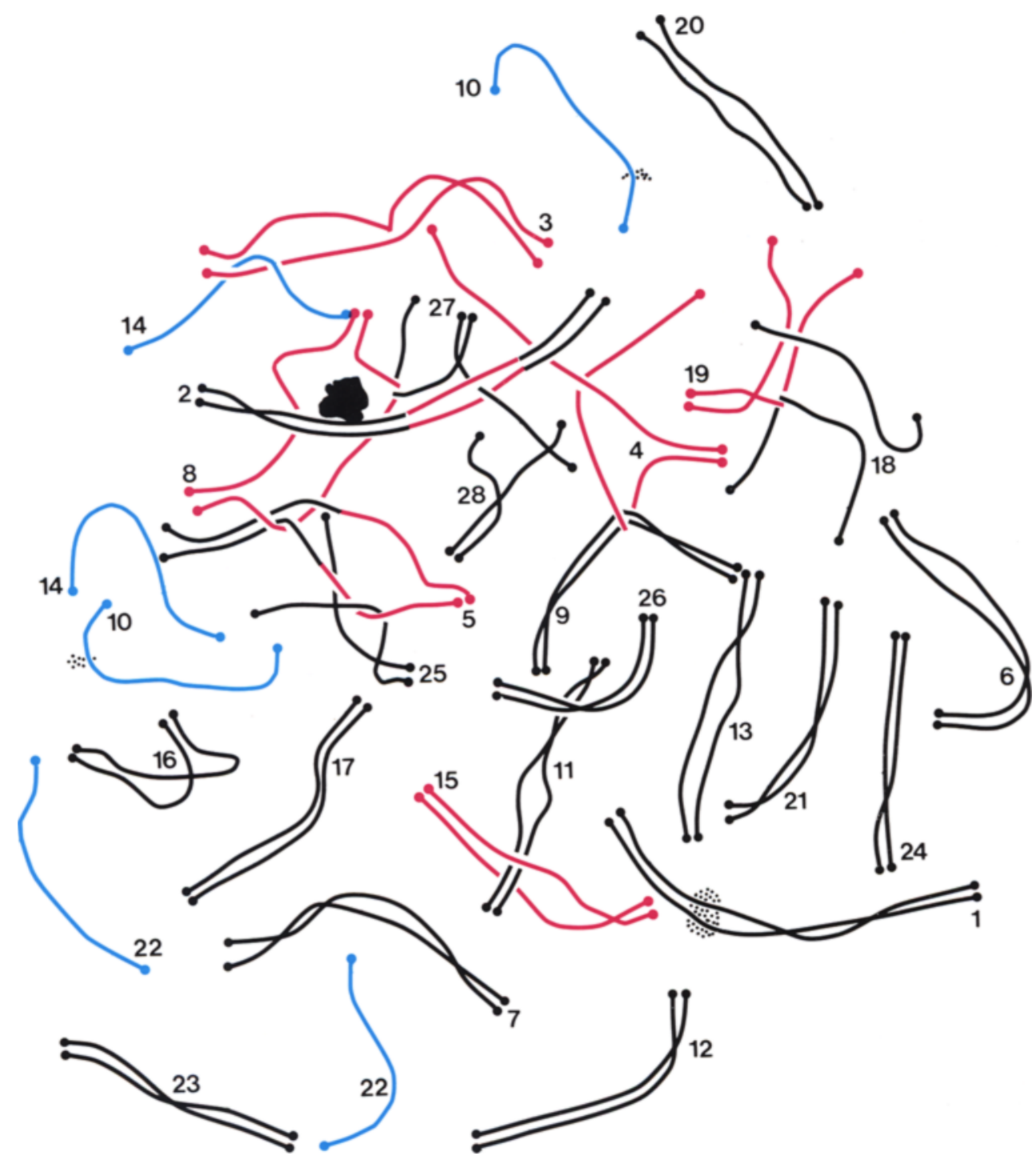

Figure 3. Spread chromosome complement at 53\% pairing. Synapsis of 26 of the 56 chromosomes is impeded by an intertwìning (4-9), a univalent interlocking (3-14), chromosome interlockings (2-27, 5-25, 8-27, 19-18) and bivalent interlockings $(8-5,15-11)$. In the tracing of the lateral components, chromosomes or chromosome segments embracing other chromosomes are shown in red and the univalents in blue. The chromosomes are numbered according to decreasing length. Chromosomes 1,2 and 10 are identified by a knob (dotted), the nucleolus organizer region (black) and a small knob (dotted), respectively. $(\mathrm{Bar}=5.0 \mu \mathrm{m})$

ure 3). Presumptive ILs not identifiable by either of these characteristics were not counted.

The central region of the $\mathrm{SC}$ responded in an unpredictable way. As a rule its presence could be inferred from the constant width of the electron transparent space between the LCs (Figures 1 and 2). A morphologically distinct central region with central component was observed in some nuclei (Figure 4). 


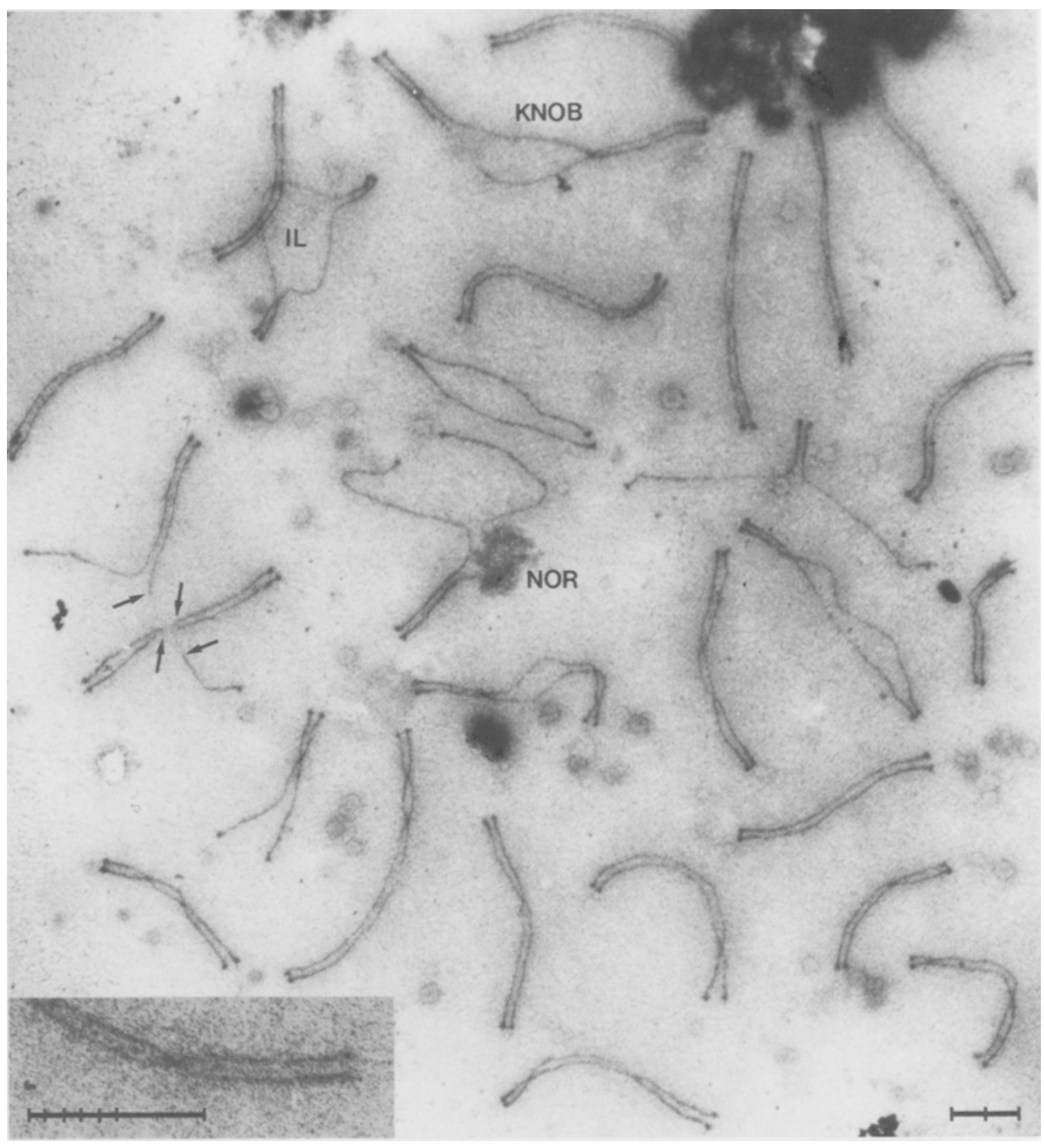

Figure 4. Spread chromosome complement at $79 \%$ pairing. Bivalent 1 is identified by the knob and bivalent 2 by the nucleolus organizer region (NOR). The central region of the synaptonemal complex is well preserved revealing at some locations a central component (insert). The nucleus contain the bivalent interlocking (IL) shown at higher magnification in Figure 1 and six free LC ends (arrows), possibly reflecting a resolving intertwining. (Bars $=2.0$ $\mu \mathrm{m})$

\subsection{The temporal sequence of zygotene and pachytene nuclei}

The sole parameter used for establishing the temporal sequence of the 270 zygotene nuclei was the extent of SC formation. Based on this criterion, the nuclei were divided into 10 groups each covering a 10 percent increment of synapsis. As can be seen in Table I the number of analysable nuclei per group increases with the extent of SC formation. This reflects the diffi- 


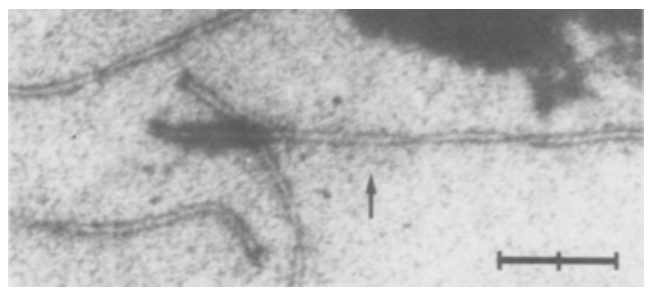

Figure 5. Bivalent 1 at pachytene stage 2 . The distal 2 $\mu \mathrm{m}$ of the lateral components have increased in width and electron density. The knob is diffuse and is not evident in this bivalent 1 (compare with Figure 26). The expected position of the knob is denoted by an arrow. $($ Bar $=2.0 \mu \mathrm{m})$

culties in obtaining well preserved complements at early zygotene stages rather than a scarcity of these stages in the material.

The temporal sequence when based exclusively on the degree of pairing is unambiguous up to late zygotene, whereas the distinction between late zygotene, in particular the $90-100 \%$ pairing interval and early pachytene (100\% pairing) although being formally well defined, may introduce bias in the determination of the frequency of ILs at these stages: Nuclei in which the only unpaired LC segments are those involved in ILs (15 of the 66 analysed nuclei) are classified as belonging to zygotene stage 10 . According to the criteria chosen for stage identification, nuclei at a similar stage of development in which ILs are absent or have been resolved are assigned to early pachytene. As a result the frequency of ILs at zygotene stage 10 is probably somewhat overestimated while the opposite is the case for early pachytene. Only 16 of the 66 nuclei at $90-100 \%$ pairing had all their unpaired LC segments involved in ILs while in the rest interlocked and free unpaired LC stretches coexist. Hence, it appears that completion of SC formation is delayed only slightly by the presence of ILs and that the determination of the IL frequency at zygotene stage 10 and early pachytene is to a minor extent biased by the ambiguity in delimiting the zygotene-pachytene transition.

Two chromosomes could be identified in nearly all nuclei, namely chromosome 1 by a knob (Figure 4) also characteristic of this chro- mosome in sectioned material (28) and chromosome 2 which carries the nucleolus organizer region (Figure 4). Apart from these two chromosomes and in some cases a second knob-bearing chromosome (Figures 3 and 26) it was not possible either in the spread or in sectioned nuclei to identify chromosomes by criteria other than their LC length.

In some late zygotene and in all pachytene nuclei, the distal $10-20 \%$ of the LC at the chromosome end closest to the knob of chromosome 1 appeared thicker and more electron dense (Figures 5 and 26). During pachytene the width of the modified LC region was 2-3 times that at zygotene and early pachytene. Similar but less pronounced modification of the $L C$ was sometimes observed in the segment between the telomere and the nucleolus organizer region in bivalent 2. Terminal modification of the LCs was occasionally observed in chromosomes other than 1 and 2 but occurred too infrequently to be of use in chromosome or stage identification. Terminal modification of the LCs has not been observed in sectioned material (28).

On the basis of the extent of terminal LC modification in bivalents 1 and 2 the 125 completely paired complements were divided into 3 groups: pachytene 1 ( 40 nuclei), pachytene 2 (67 nuclei) and pachytene 3 (18 nuclei).

\subsection{Synapsis and SC formation}

Spreading of the chromatin followed by silver staining of the LCs reveal continuous LCs at leptotene and very early zygotene. Nuclei with $0-10 \%$ pairing contain thin but apparently continuous LCs and at $10-20 \%$ pairing, the LCs appear distinct with well differentiated terminal thickenings in the best preserved complements (Figure 6). The initial recognition between homologous chromosomes can be monitored with great accuracy in such nuclei.

Two different types of association between chromosomes are evident prior to SC formation. In the first type, contact is established between short regions located $1-3 \mu \mathrm{m}$ from the telomeres (Figures 6 and 7). The distance between the involved homologous chromosome segments is several times the width of the completed SC. The segments distal to the contact 


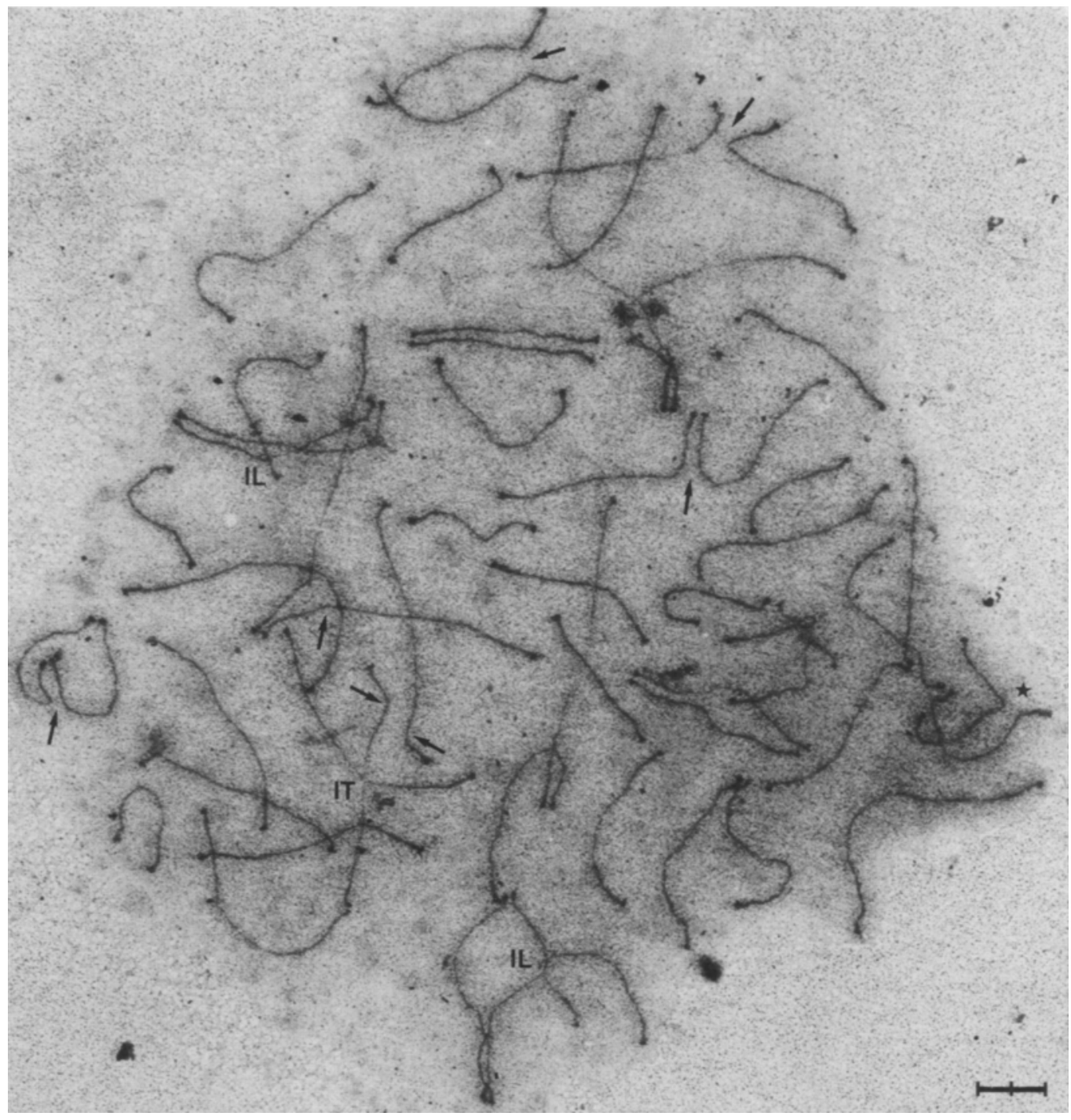

Figure 6. Spread chromosome complement at $11 \%$ pairing. All 56 chromosomes can be traced by their continuous lateral components extending from telomere to telomere. Associations between subtelomeric sites of homologous chromosomes are denoted by arrows and an association between supposedly nonhomologous sites is indicated by a star. Synaptonemal complex formation is nearly completed in one bivalent while the SC only extends from the telomeres to the presumptive subtelomeric association sites in the partially paired bivalents. Two univalents are interlocked $(\mathrm{IL})$ between the homologues of interstitially unpaired bivalents. IT, possible intertwining. (Bar $=2.0$ $\mu \mathrm{m})$

region often diverge, indicating absence of more distal contact regions of this type. When SC formation has occurred it is nearly always in the region between the subtelomeric association sites and the nearest telomere (Figures 6 and 7).
SC formation in the opposite direction is rare. Among more than 3,500 partially paired bivalents very few were observed in which SC formation had occurred interstitially leaving both ends unpaired (Figure 7). With few exceptions, the 

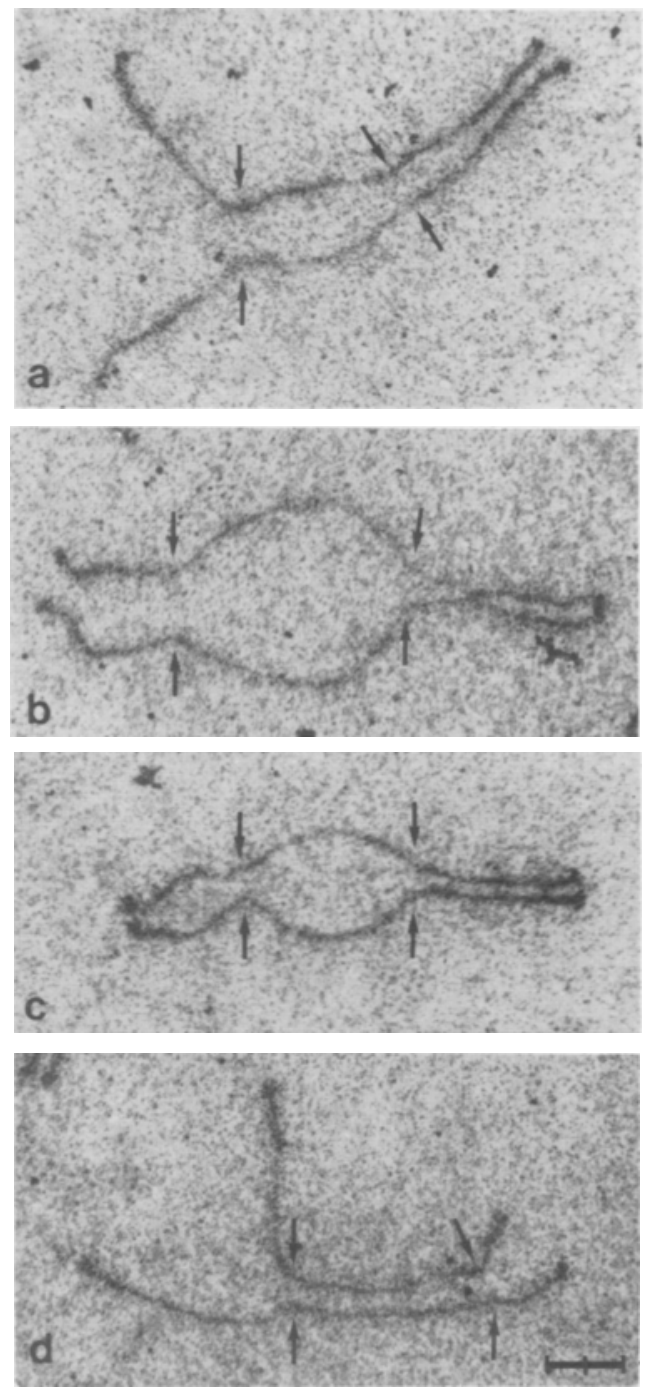

Figure 7. Examples of synapsing homologues. In Figure 7a the homologues are held together by an SC segment at one end but are only associated at the subtelomeric sites at the other end. The distance between the subterminal association sites is $2-3$ times the width of the central region of the fully formed SC. The homologues in Figure $7 \mathrm{~b}$ are paired with an SC at one end and are associated subterminally at the other end, the telomeres being close together. The bivalent in Figure $7 \mathrm{c}$ is similar to that in Figure $7 \mathrm{~b}$ except that SC formation has just commenced from the telomeres near the subterminal association sites. Figure $7 \mathrm{~d}$ shows one of the rare examples of interstitial SC formation leaving the telomeres unpaired at both ends. The presumptive positions of the subtelomeric association sites are indicated by arrows. (Bar $=1.0 \mu \mathrm{m}$ )

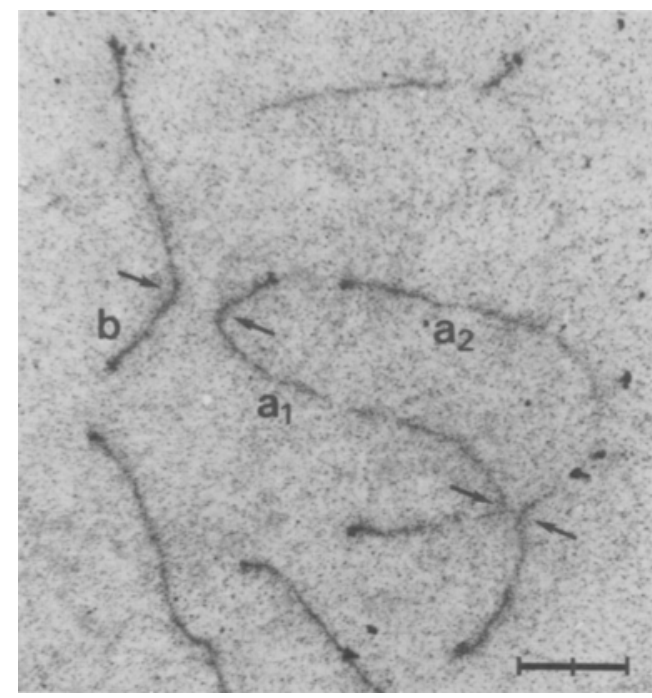

Figure 8. Association between subtelomeric sites (arrows) of two chromosomes of equal length $\left(a_{1}\right.$ and $\left.a_{2}\right)$. At the opposite end $a_{1}$ is associated with a third, shorter chromosome (b). The latter two subtelomeric association sites are located at different distances from the telomeres while the association sites of $a_{1}$ and $a_{2}$ are equidistant. Apparently, the $a_{1}-a_{2}$ association is homologous whereas the $\mathrm{a}_{1}$-b association is not. $(\mathrm{Bar}=2.0$ $\mu \mathrm{m})$

subterminal association sites are located equidistant from the telomeres in LCs of equal lengths, i.e., they are probably homologous regions. Subterminal associations at one or both ends of a pair of homologues are frequent in the first period of zygotene and always appear to precede SC formation between homologues. Images like those shown in Figures 6 and 7 suggest that the subterminal associations may not be directly related to the initiation of SC formation. In Figures $7 b$ and $c$ for example, SC formation appears to start from the telomeric plaques rather than from the subterminal association sites.

Associations of this type are rarely observed between nonhomologous chromosomes. The three LCs in Figure 8 comprise a pair of homologues $\left(a_{1}\right.$ and $\left.a_{2}\right)$ associated subterminally and a third chromosome (b) associated nonhomologously with one member $\left(a_{1}\right)$ of the pair. A second example of a nonhomologous primary association is shown in Figure 6. 


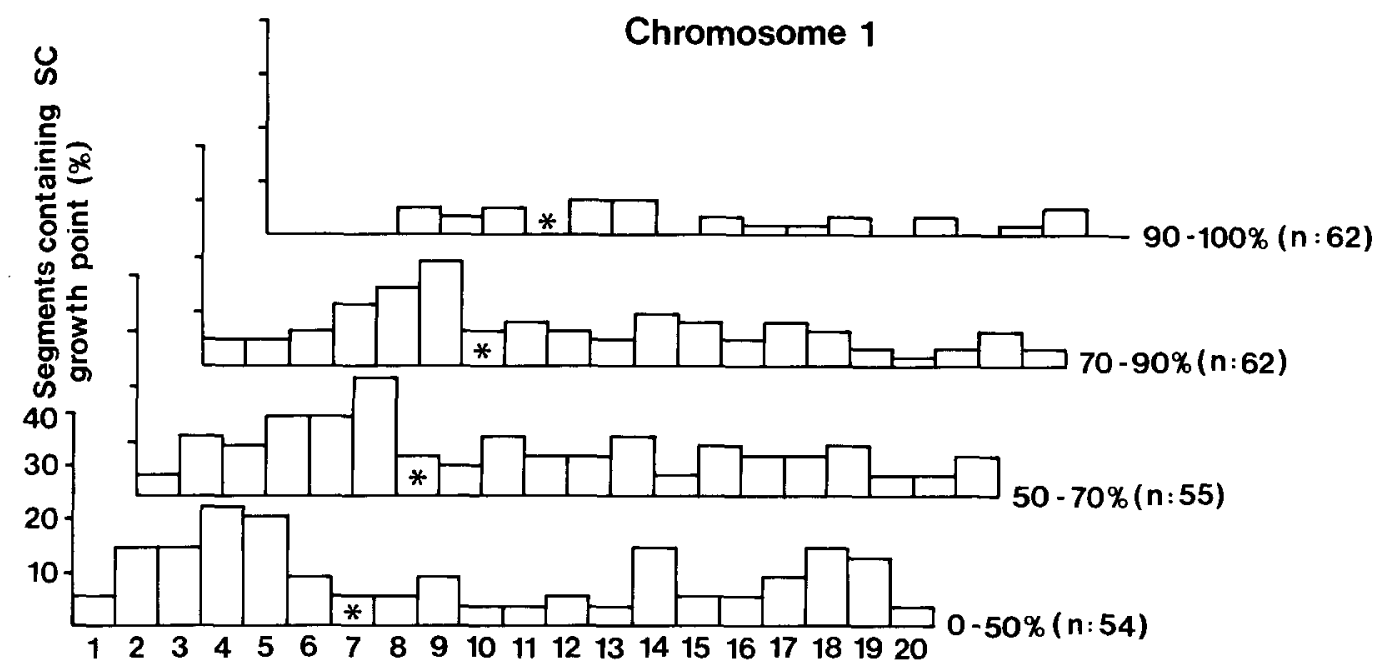

Figure 9. Histograms showing the distribution of synaptonemal complex end points along bivalent 1 at $0-50 \%$, $50-70 \%, 70-90 \%$ and $90-100 \%$ pairing. The mean lateral component length of each bivalent was normalized to the mean length of all bivalents 1 at zygotene. The normalized lengths were divided into 20 intervals and the percentage of segments containing an SC end point was determined for each interval. The mean position of the knob is denoted by an asterisk. $n$, number of observations.

In an attempt to identify the position of the primary association sites in individual chromosomes, the location of $\mathrm{SC}$ end points during zygotene was determined for bivalents 1 and 2 , the only two bivalents which could be identified and oriented by morphological markers. The LC lengths were normalized to their mean length at zygotene and divided into 20 segments (each about $0.5 \mu \mathrm{m})$. The zygotene nuclei were divided into four groups $(0-50 \%, 50-70 \%, 70-90 \%$ and

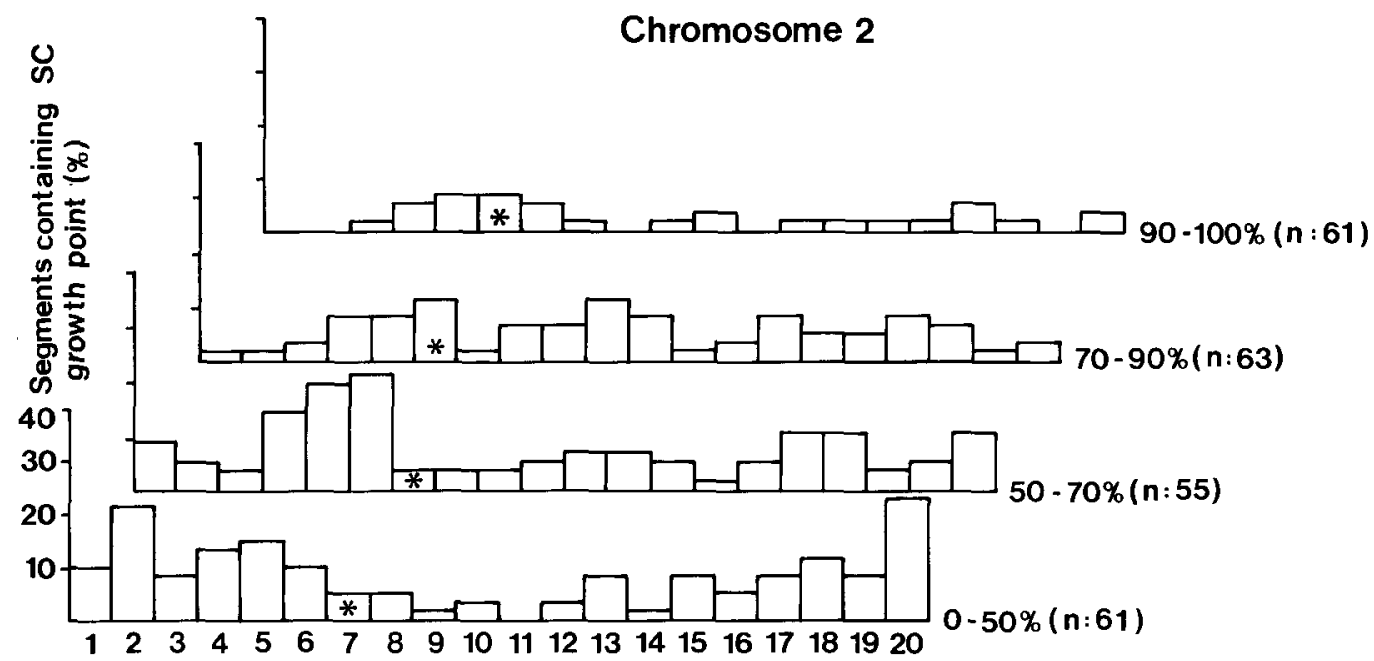

Figure 10. Histogram showing the distribution of synaptonemal growth points along bivalent 2 at $0-50 \%, 50-70 \%$, $70-90 \%$ and $90-100 \%$ pairing. For further details, see legend to Figure 9 . The position of the nucleolus organizer region is indicated by an asterisk. 


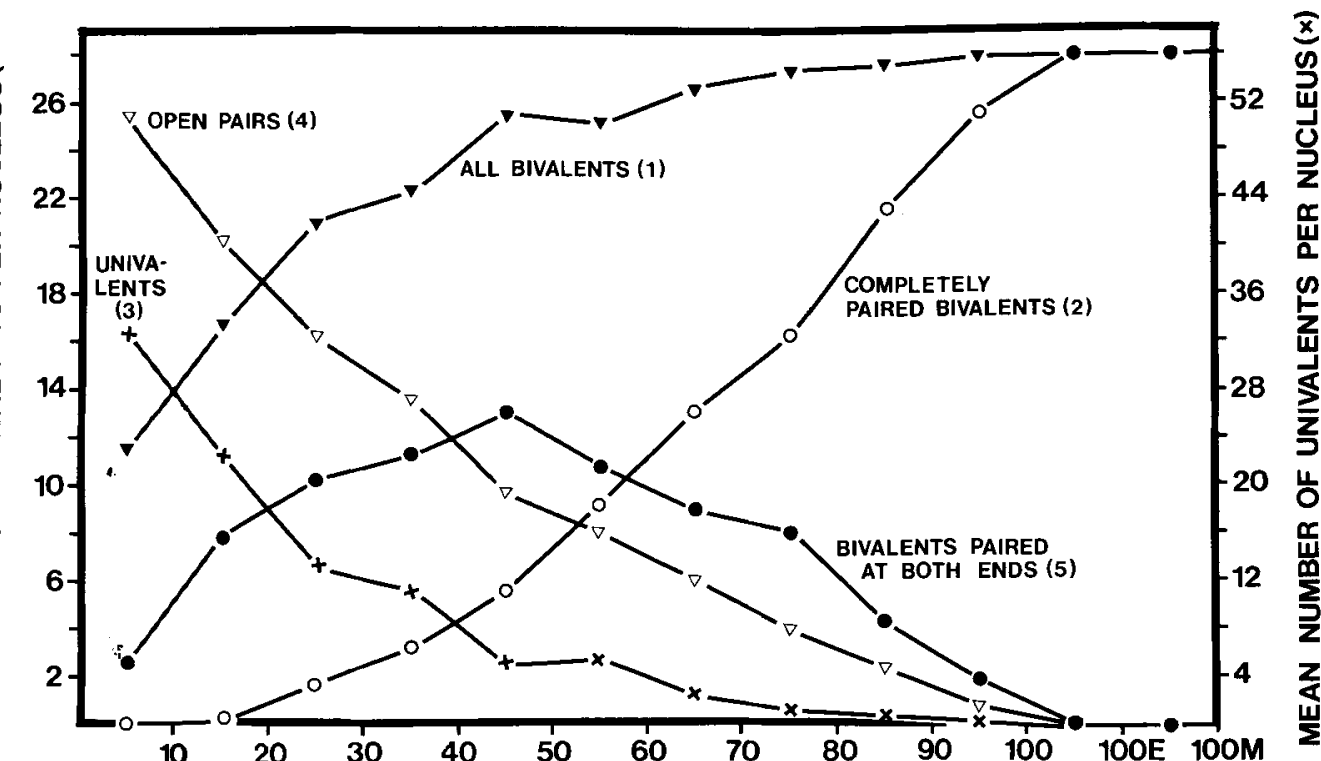

\begin{tabular}{|c|c|c|c|c|c|c|c|c|c|c|c|c|}
\hline $\begin{array}{l}\text { Number } \\
\text { of nuclei }\end{array}$ & 5 & 10 & 17 & 16 & 15 & 28 & 33 & 39 & 40 & 66 & 40 & 67 \\
\hline $\begin{array}{l}\text { Mean \% } \\
\text { pairing } \\
\text { SO }\end{array}$ & $\begin{array}{l}7.4 \\
3.5\end{array}$ & $\begin{array}{r}13.2 \\
3.0\end{array}$ & $\begin{array}{r}24.5 \\
3.4\end{array}$ & $\begin{array}{r}35.0 \\
3.1\end{array}$ & $\begin{array}{r}44.5 \\
2.9\end{array}$ & $\begin{array}{r}55.0 \\
3.2\end{array}$ & $\begin{array}{r}64.7 \\
3.3\end{array}$ & $\begin{array}{r}75.5 \\
2.9\end{array}$ & $\begin{array}{r}85.7 \\
3.0\end{array}$ & $\begin{array}{r}95.3 \\
2.5\end{array}$ & $\begin{array}{l}100 \\
-\end{array}$ & $\begin{array}{l}100 \\
-\end{array}$ \\
\hline $\begin{array}{l}\text { (v) SD } \\
\text { Range }\end{array}$ & $\begin{array}{l}4.5 \\
3-16\end{array}$ & $\begin{array}{c}2.8 \\
10-19\end{array}$ & $\begin{array}{c}2.7 \\
14-25\end{array}$ & $\begin{array}{c}2.9 \\
16-27\end{array}$ & $\begin{array}{c}3.3 \\
15-27\end{array}$ & $\begin{array}{c}1.6 \\
21-28\end{array}$ & $\begin{array}{c}1.2 \\
24-28\end{array}$ & $\begin{array}{c}1.0 \\
25-28\end{array}$ & $\begin{array}{c}0.6 \\
26-28\end{array}$ & $\begin{array}{c}0.1 \\
27-28\end{array}$ & 28 & $\overline{28}$ \\
\hline $\begin{array}{l}\text { (O) SD } \\
\text { Range }\end{array}$ & $\begin{array}{l}0 \\
0\end{array}$ & $\begin{array}{l}0.6 \\
0-2\end{array}$ & $\begin{array}{l}1.3 \\
0-3\end{array}$ & $\begin{array}{l}1.8 \\
0-6\end{array}$ & $\begin{array}{l}2.6 \\
1-10\end{array}$ & $\begin{array}{l}1.8 \\
6-12\end{array}$ & $\begin{array}{l}2.6 \\
8-17\end{array}$ & $\begin{array}{c}1.7 \\
13-21\end{array}$ & $\begin{array}{c}1.7 \\
18-25\end{array}$ & $\begin{array}{c}1.3 \\
21-27\end{array}$ & $2 \overline{8}$ & $2 \overline{8}$ \\
\hline $\begin{array}{l}(x) \text { SD } \\
\text { Range }\end{array}$ & $\begin{array}{c}9.0 \\
24-50\end{array}$ & $\begin{array}{c}5.5 \\
18-36\end{array}$ & $\begin{array}{l}5.5 \\
6-28\end{array}$ & $\begin{array}{l}5.8 \\
2-24\end{array}$ & $\begin{array}{l}2.7 \\
2-10\end{array}$ & $\begin{array}{l}3.1 \\
0-14\end{array}$ & $\begin{array}{l}2.4 \\
0-8\end{array}$ & $\begin{array}{l}1.9 \\
0-6\end{array}$ & $\begin{array}{l}1.1 \\
0-4\end{array}$ & $\begin{array}{l}0.2 \\
0-2\end{array}$ & $\overline{0}$ & $\overline{0}$ \\
\hline $\begin{array}{l}(\nabla) \text { So } \\
\text { Range }\end{array}$ & $\begin{array}{c}1.0 \\
24-27\end{array}$ & $\begin{array}{c}2.0 \\
17-23\end{array}$ & $\begin{array}{l}3.2 \\
7-21\end{array}$ & $\begin{array}{c}2.4 \\
10-17\end{array}$ & $\begin{array}{l}2.0 \\
5-13\end{array}$ & $\begin{array}{l}2.3 \\
4-13\end{array}$ & $\begin{array}{l}2.0 \\
2-10\end{array}$ & $\begin{array}{l}2.0 \\
1-8\end{array}$ & $\begin{array}{l}1.5 \\
0-6\end{array}$ & $\begin{array}{l}0.8 \\
0-3\end{array}$ & $\overline{0}$ & $\overline{0}$ \\
\hline $\begin{array}{l}\text { (e) SD } \\
\text { Range }\end{array}$ & $\begin{array}{l}1.0 \\
2-4\end{array}$ & $\begin{array}{l}2.0 \\
5-11\end{array}$ & $\begin{array}{l}3.8 \\
4-21\end{array}$ & $\begin{array}{l}3.4 \\
6-18\end{array}$ & $\begin{array}{l}3.3 \\
7-20\end{array}$ & $\begin{array}{l}3.3 \\
6-20\end{array}$ & $\begin{array}{l}3.6 \\
2-17\end{array}$ & $\begin{array}{l}2.4 \\
3-11\end{array}$ & $\begin{array}{l}2.1 \\
1-9\end{array}$ & $\begin{array}{l}1.2 \\
0.4\end{array}$ & $\overline{0}$ & $\overline{0}$ \\
\hline
\end{tabular}

Figure 11. Kinetics of synapsis in Bombyx spermatocytes. Percent pairing of lateral components into synaptonemal complex is shown on the abscissa, $100 \mathrm{E}$ (early) and $100 \mathrm{M}(\mathrm{mid})$ indicating pachytene stages 1 and 2 . The graphs show the frequency of 1: partially and fully paired bivalents, 2: completely paired bivalents, 3 : univalents, 4: bivalents with only one SC segment and univalents, i.e., the total number of pairs of homologues capable of interlocking other chromosomes and 5: bivalents with an SC segment at both ends and an unpaired middle region.

The table below the diagram includes in addition to the number of nuclei and the mean percent pairing at each substage, the standard deviation (SD) and the range of the mean values shown graphically.

$90-100 \%$ pairing) and the percentage of segments containing an end point for $\mathrm{SC}$ formation was determined (Figures 9 and 10). The two histograms show, especially clearly for bivalent
1, that there is a markedly higher frequency of segments with an SC end point subterminally in the short "arm" than in the remaining part of the bivalent. This indicates that the knob in bivalent 

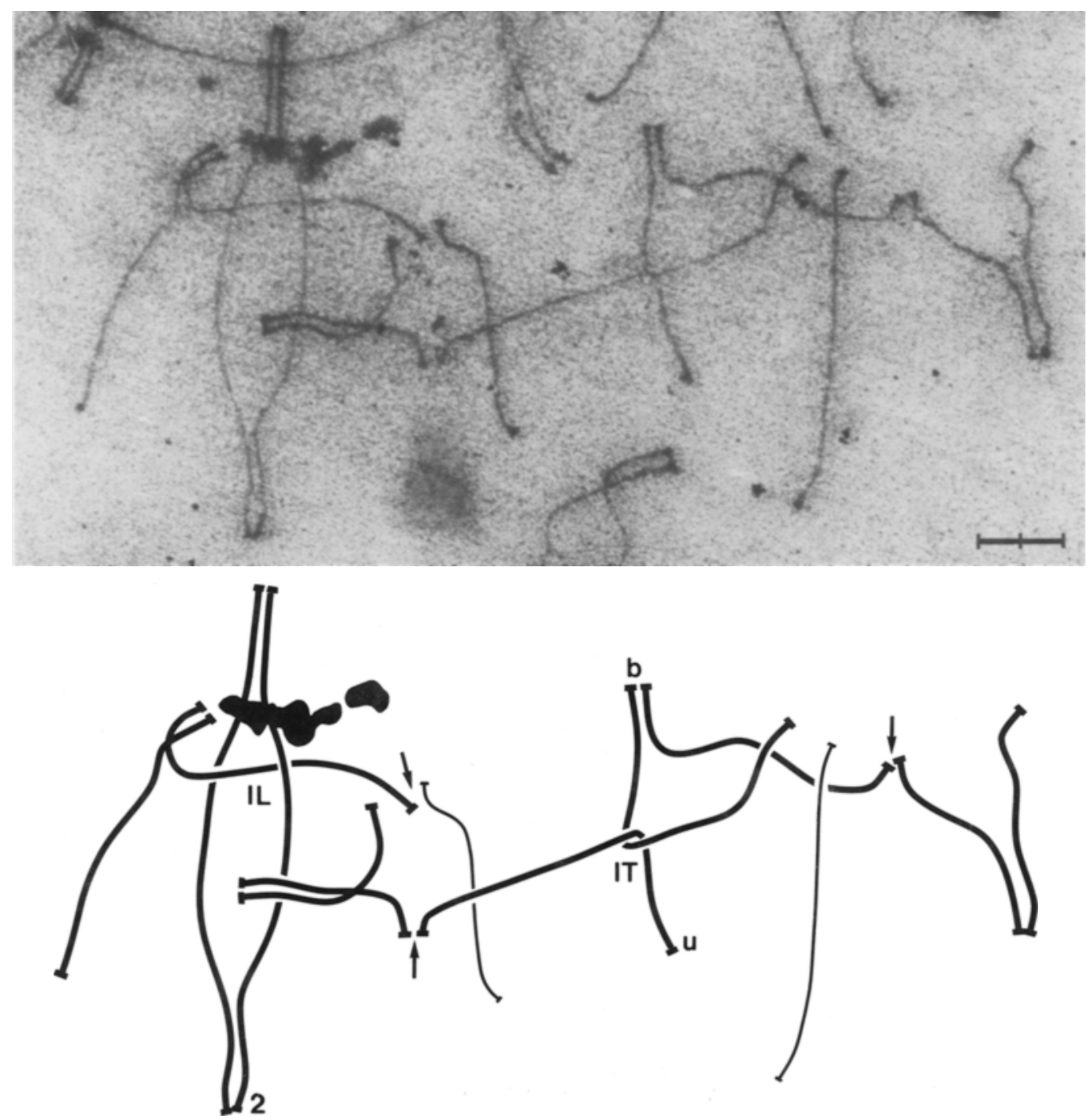

Figure 12. Partially paired bivalents and univalents from a nucleus at $39 \%$ pairing. Completed synaptonemal complex is only present between the presumptive subtelomeric initiation sites and the nearest telomeres in the par̀tially paired bivalents. Associations involving only the telomeric plaques are observed at several places (arrows), in all cases between supposedly nonhomologous chromosomes. One interlocking (IL) involving bivalent 2 and an intertwining (IT) of a univalent ( $\mathrm{u}$ ) and one homologue of a partially paired bivalent are indicated in the drawing. $($ Bar $=2.0 \mu \mathrm{m})$

1 and the nucleolus organizer region in bivalent 2 represent an impediment to synapsis and SC formation and hence increase the frequency of partially paired bivalents 1 and 2 with SC end points close to the morphological markers. A similar delay in $\mathrm{SC}$ formation has previously been noted for the centromeres and secondary constrictions of human zygotene bivalents (44). The weak effect of subterminal associations on the distribution of SC end points in the long arms of bivalents 1 and 2 is probably due to extensive asynchrony of synapsis even among 


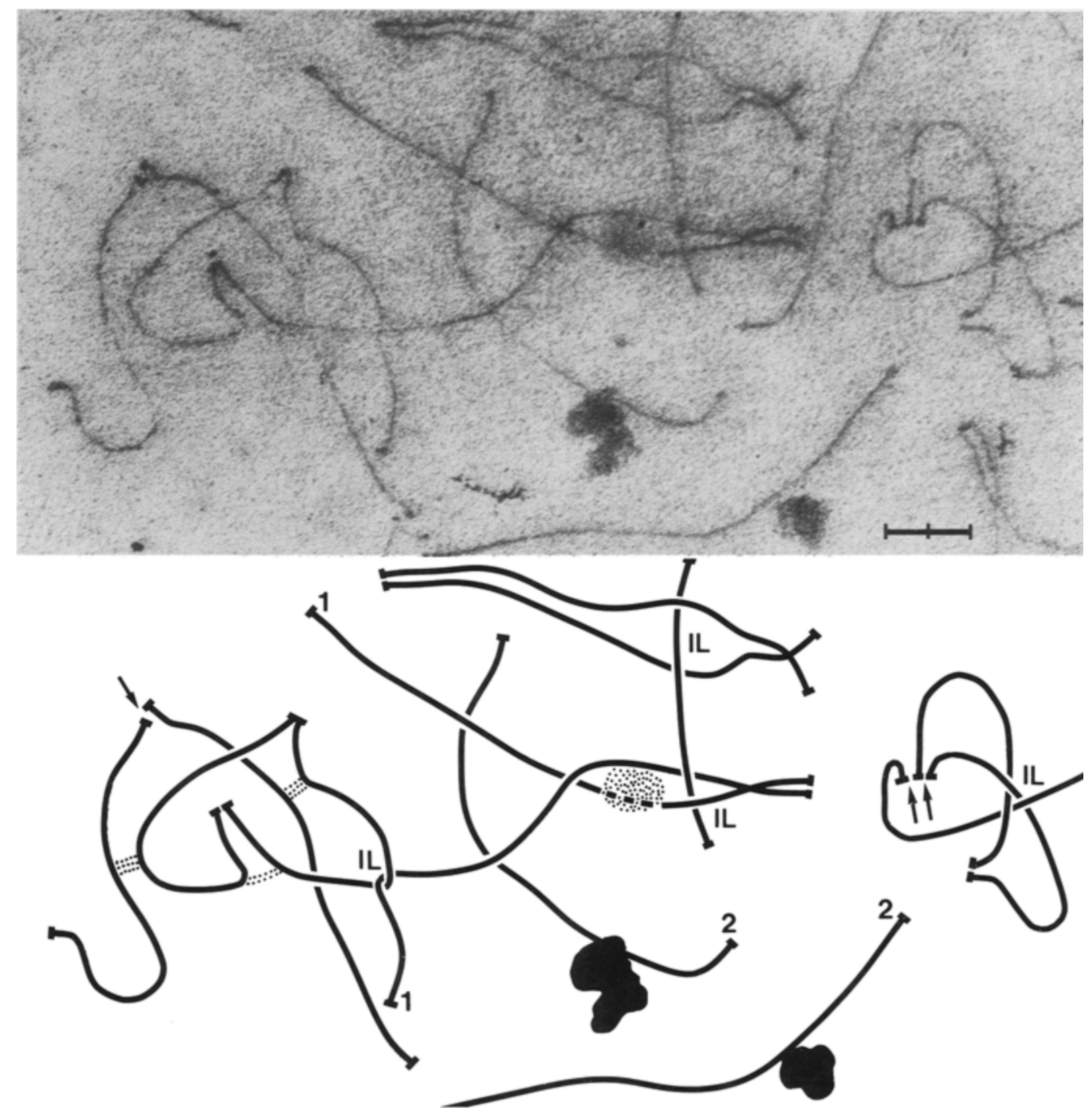

Figure 13. Partially paired bivalents and univalents from a nucleus at $24 \%$ pairing. Telomeric associations are indicated by arrows. Presumptive associations between interstitial sites of nonhomologous chromosomes are indicated by rows of dots in the drawing. IL, interlocking; 1,2 , bivalents 1 and 2 . (Bar $=2.0 \mu \mathrm{m})$

nuclei at the same stage of pairing (see Figure 11).

It is evident from Figure 11 that synaptic initiation occurs preferentially during the first half of zygotene. At a time when $40-50 \%$ of the complement is paired with an SC, a mean of 51.6 LCs or $92 \%$ of all chromosomes (Figure 11 , curve 1) form bivalents of which 18.4 are partially paired and 7.4 fully paired (curve 2). Only a mean of 4.4 LCs are still unpaired (curve 3). Curve 4 in Figure 11 depicts the mean frequency of "open" bivalents, i.e. homologues still capable of interlocking other chromosomes. This frequency gradually declines as synapsis progresses, but still amounts to approximately 9 at $40-50 \%$ pairing and to about 2 at $80-90 \%$ pairing, which shows that interfocking in principle is possible during most of the zygotene stage. 


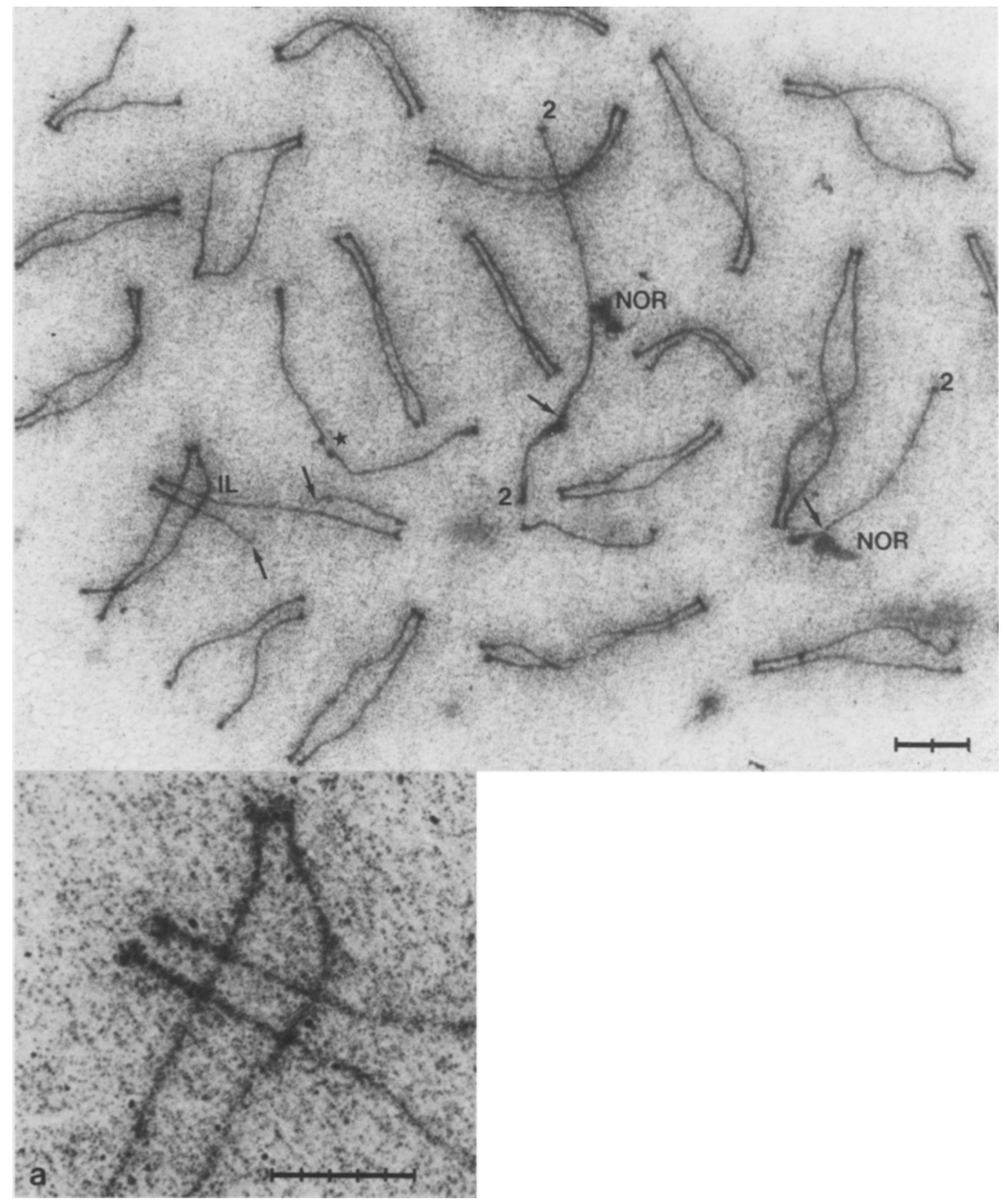

Figure 14. Synapsing chromosomes from a spread nucleus at 53\% pairing. Most of the partially paired bivalents have a short SC segment at one or both ends. An association involving only the telomeric plaques of two otherwise unassociated homologues is denoted by a star. One of the unpaired chromosomes 2 which carries a small dense nucleolus (NOR) is broken at the nucleolus organizer region (arrows), the shorter segment lying at the telomeric plaque of the intact chromosome 2 . A second LC discontinuity (arrows) is apparent in the middle unpaired region of a partially paired bivalent. ( $\mathrm{Bar}=2.0 \mu \mathrm{m})$. The crossing $\mathrm{LCs}$ of the presumptive interlocking (IL) are shown at higher magnification in Figure 14a. $($ Bar $=1.0 \mu \mathrm{m})$ 


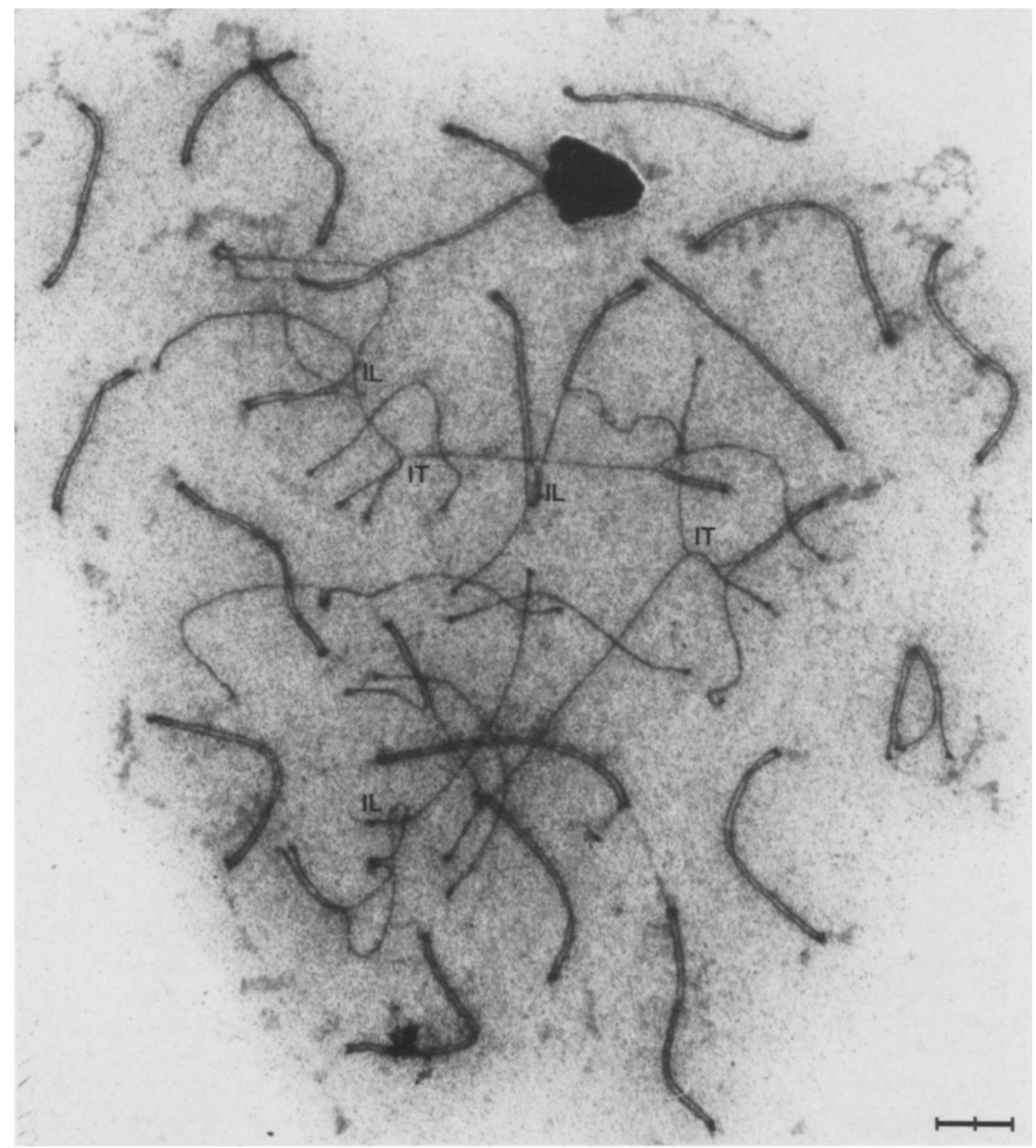

Figure 15. Spread chromosome complement from a nucleus at $65 \%$ pairing. IL, interlocking; IT, intertwining. (Bar $=2.0 \mu \mathrm{m}$ )

As can be seen from curve 5 in Figure 11, about $50 \%$ of the partially paired bivalents have an SC segment at both ends in complements from 10 to $80 \%$ paired. The SC segments in such bivalents, as well as those of partially paired bivalents with only one SC segment are usually short and of about equal length (1-3 $\mu \mathrm{m})$ (Fig- ures 6 and 7). This is not consistent with a constant rate of $\mathrm{SC}$ formation but rather suggests that there is a temporal delay/arrest of SC formation after the initial subterminal association has been stabilized by SC formation in the interval to the nearest telomeres.

It is thus likely that the primary recognition 


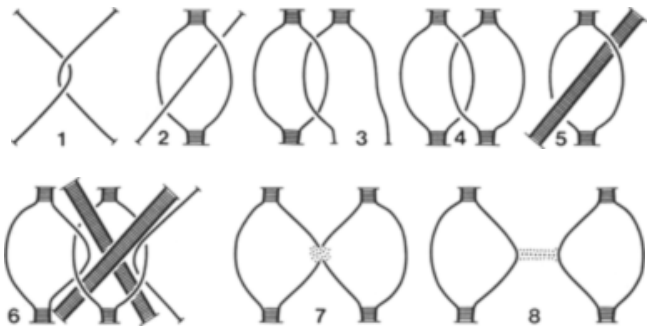

Figure 16. Diagram defining the different types of entanglements encountered in spread zygotene complements. 1) Intertwining. 2) Univalent interlocking. 3 , 4) Interlocking of one homologue of a partially paired bivalent - chromosome interlocking. 5) Interlocking of a partially or completely paired bivalent bivalent interlocking. 6) Multiple interlocking. This category includes all cases in which more than two pairs of homologues are involved. 7,8 ) Resolving interlocking.

During synapsis the telomeres remain attached to the inner nuclear membrane (34) and only intertwining of chromosomes (type 1) are resolvable via movements of the telomeres on the membrane. Resolution of all other types of entanglements requires breakage and reunion of both sister chromatids of one or more of the involved chromosomes.

between homologous chromosomes is effected by short segments of the chromosomes located subterminally in the chromatin. Subsequent SC formation starts frequently but not exclusively from closely associated attachment plaques of the telomeres and progresses towards the nearest subterminal association site. After a delay at the association sites, completion of the $\mathrm{SC}$ in a bivalent occurs quite rapidly, if not impeded by interlocking.

A second type of chromosome association preceding $\mathrm{SC}$ formation is frequently formed between the dense attachment plaques of two or more LCs. (Figures 12 and 13). Associations of this type are infrequent at early zygotene but become more common as synapsis progresses. In contrast to the apparently specific subterminal associations, these terminal associations hold the LCs at a distance similar to that of the central region of the SC and involve homologous as well as nonhomologous chromosomes (Figures 12, 13 and 14).

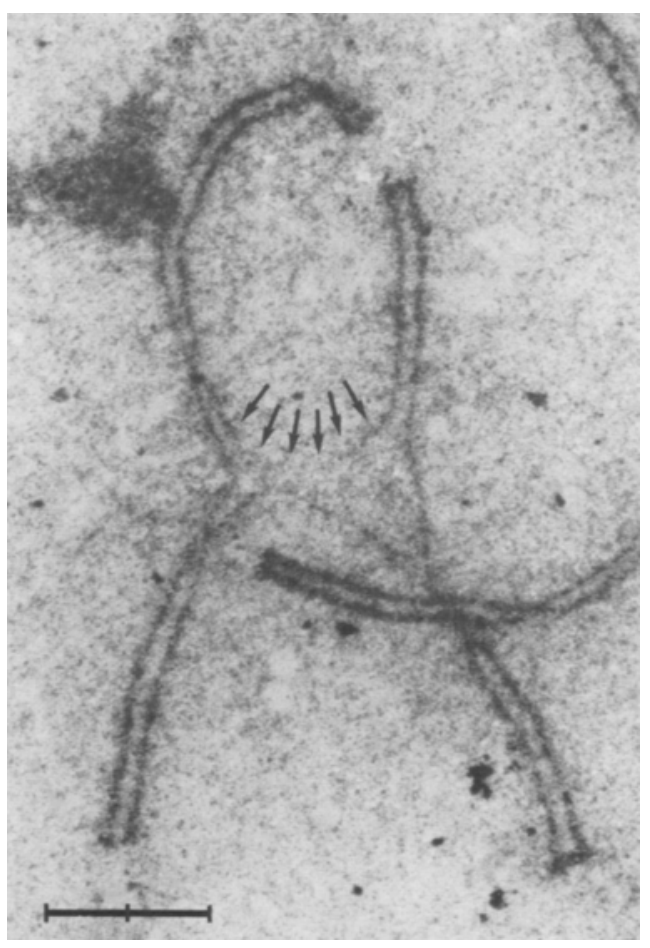

Figure 17. Resolving bivalent interlocking (arrows). $($ Bar $=2.0 \mu \mathrm{m})$

The telomeric associations also differ from subterminal ones by often involving more than two chromosomes as shown in Figure 13, where a telomere of a single $\mathrm{LC}$ is associated with the paired telomeres of a bivalent. The micrograph and the interpretative drawing in Figure 12 show that the terminal association is not simply due to fortuitous location of telomeres after spreading: The univalent $(\mathrm{u})$ has been under tension during spreading and although one LC of bivalent $b$ is bent and the univalent stretched due to the intertwining of the two chromosomes, the terminal association is maintained.

\subsection{Interlocking and resolution}

It is obvious from Figures 3, 12, 13 and 15 that homologous chromosomes prior to zygotene are not so located that entanglements are avoided, even when allowance is made for possible intertwining of chromosomes when the three-dimensional architecture of the nucleus is disrupted by 


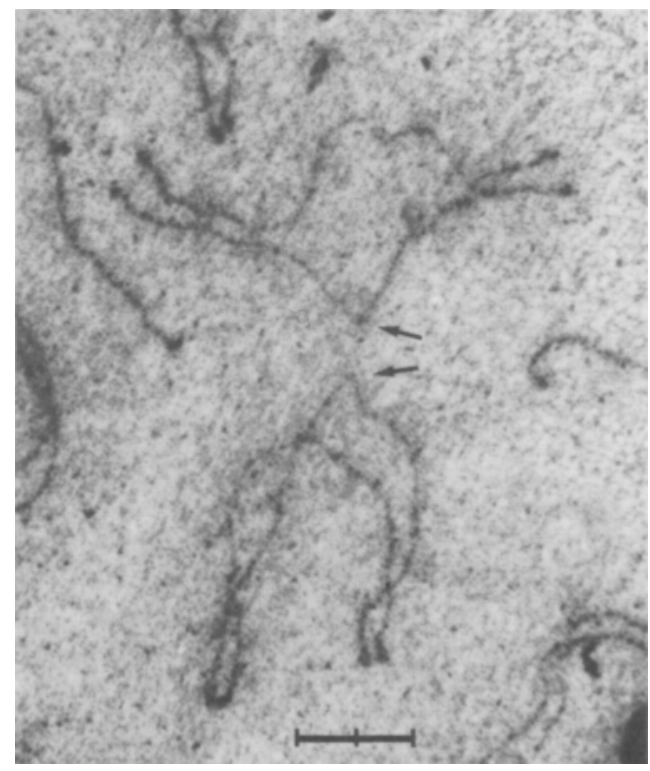

Figure 18. Resoiving chromosome interlocking (arrows). (Bar $=2.0 \mu \mathrm{m}$ )

the spreading of the chromosome complement. Entanglements in the form of interlocking and breaks were found in $87 \%$ of all analysed zygotene nuclei. The diagram in Figure 16 depicts the different categories of entanglements distinguished and describes how they are referred to below. The micrographs and the accompanying interpretative drawings in Figures $3,12,13,17,18,19$ and 22 illustrate their appearance.

The frequency of IL events (single, multiple and resolving ILs) as well as number of univalents and partially and fully paired bivalents involved in each IL event was determined for each of the 10 zygotene substages. Curve 1 in Figure 20 shows the variation in the mean total number of ILs during zygotene. Despite the irregular shape of the curve (most likely because the number of nuclei analysed is insufficient to compensate for the internuclear variation as indicated in the tables of Figures 11,20 and 21), it is evident that ILs are present throughout the zygotene stage. Nuclei $0-10 \%$ paired contain a mean of 1.2 ILs, nuclei 10-90\% paired between 1.9 and 3.4 ILs and at $90-100 \%$ pairing a mean
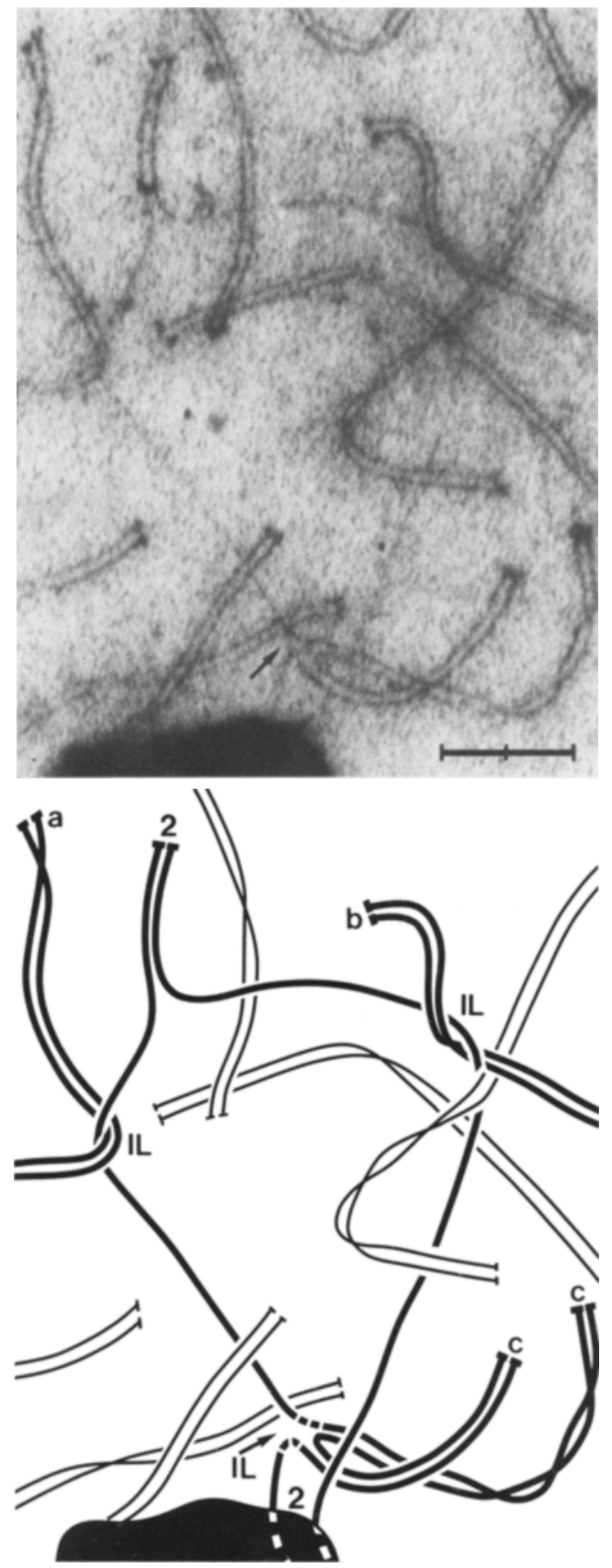

Figure 19. Multiple interlocking. The interstitially unpaired lateral components of bivalent 2 interlock 3 bivalents ( $a, b$ and $c)$. The interlocked bivalent $c$ appears to be in the process of being released. The LC of one homologue has passed the interlocking chromosome 2 while the second LC is confluent with the LC of chromosome 2 (arrow). The interpretation is shown diagrammatically below. $(\mathrm{Bar}=2.0 \mu \mathrm{m})$ 


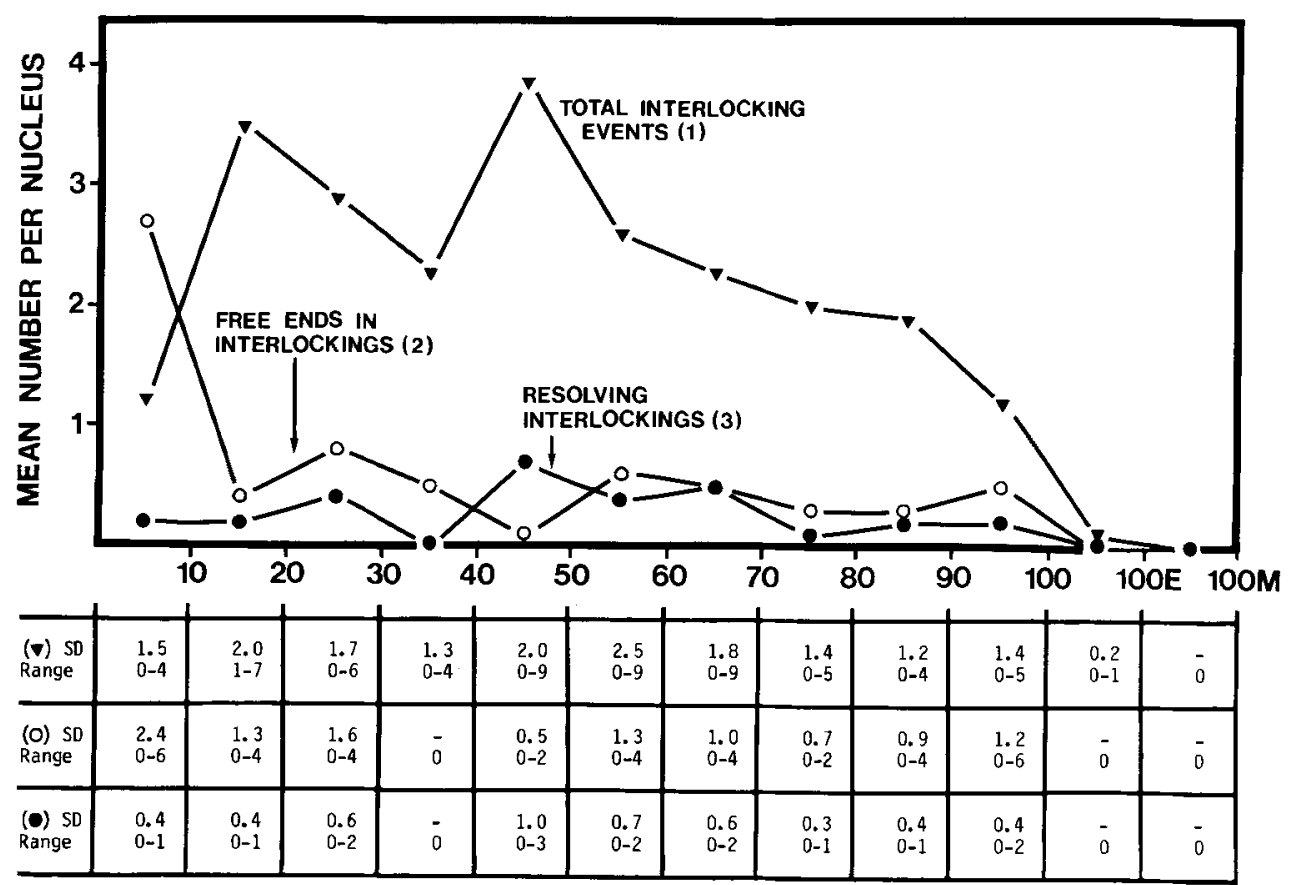

Figure 20. The frequency of 1 : interlocking events, 2 : free lateral component ends in presumptive interlockings and 3: resolving interlockings during the 10 substages of zygotene and pachytene stages 1 (100-100E) and 2 (100E-100M).

The table includes the standard deviation (SD) and the range of the mean values shown graphically.

of 1.2 ILs are detected. The range and standard deviation of the numbers on which the curve is based are large, indicating considerable variation among nuclei of the same substage. The mean number of chromosomes affected by IL events, either by actually being interlocked, by having its homologue interlocked or by interlocking other chromosomes, varies between 8 and 13 in nuclei $20-90 \%$ paired (Figure 21 , curve 1 .).

Interlocking thus constitutes a major obstacle to regular synapsis of a considerable fraction of the chromosome complement. The frequencies of interlocked univalents (type 2, Figure 16) and of one homologue of a partially paired bivalent (types 3 and 4, Figure 16) are illustrated by curves 2 and 3 in Figure 21. The mean number of interlocked univalents increases early in zygotene and declines rapidly as pairing increases from 10 to $30 \%$. The mean number of interlocked homologues of partially paired bivalents, types 3 and 4, exhibits a slower increase reaching a maximum at $30-50 \%$ pairing followed by a gradual decrease as synapsis progresses. The mean number of interlocked bivalents on the other hand increases from zero almost linearly up to $90 \%$ pairing reaching a mean of about 2 per nucleus. Between 90 and 100\% pairing, all interlocked bivalents are released. The number of multiple ILs per nucleus is low throughout synapsis ranging between 0.1 and 0.7 , most of the substages having mean frequencies of 0.3 to 0.4 .

It is evident from curves 2, 3 and 4 in Figure 21 that ILs involving a univalent (type 2), one homologue of a bivalent (types 3 and 4) and to a lesser extent bivalent ILs (type 5) are continuously resolved as synapsis progresses. Theoretically, new ILs can form as long as "open" pairs of homologues are present in the nucleus (curve 4 , Figure 11 ), i.e. nearly up to $100 \%$ pairing. It is thus likely that the mean frequencies upon which curves 1-4 in Figure 21 as well as curve 1 in Figure 20 are based, express the combined 


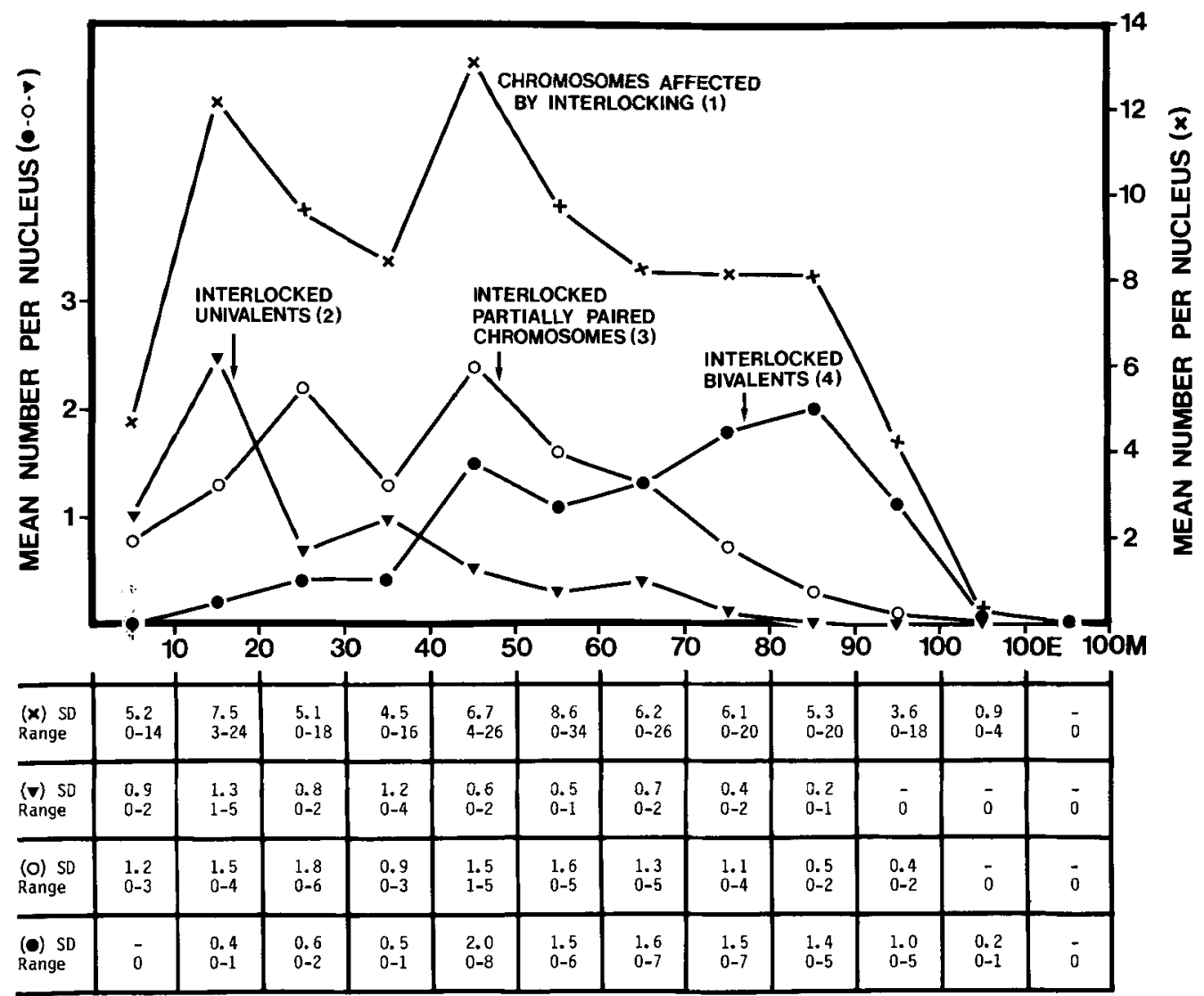

Figure 21 . The frequency of 1 : chromosomes affected by interlocking (by interlocking other chromosomes, by being interlocked or by having its homologue interlocked), 2: interlocked univalents, 3 : interlocked homologue of a partially paired bivalent - chromosome interlocking and 4: interlocked homologues of a bivalent - bivalent interlocking.

The table includes the standard deviation (SD) and the range of the mean values shown graphically.

contribution of formation and resolution of ILs. Therefore, the actual number of ILs occurring in an average nucleus probably exceeds the mean frequencies, determined from the spread material.

In a number of instances, entanglements of type 1 (Figure 16) were observed (Figure 15). Although untangling of intertwined chromosomes is theoretically possible without chromosome breakage, the intertwining represents a restraint to chromosome movement identical to that of the regions of contact between interlocked chromosomes and may accordingly be detected and resolved by the same break-repair system which normally resolves true IL (Figures 4 and 6).
Analysis of the break-repair resolution process is hampered by the fact that the chromosomes are represented only by their LC, and thus processes occurring in the chromatin cannot be observed directly. Passage of two chromosomes therefore has to be inferred from the morphology of the two passing LC segments. In a number of cases (those counted and graphically shown in curves 2 and 3 in Figure 20), free ends of LCs were observed in situations where an IL appeared to be resolving (Figure 22). Additional free LC ends (mean frequency for all zygotene stages $=0.1$; range $=0-0.3$ ) were observed in chromosomes not obviously involved in ILs (Figure 14). The latter category probably includes free LC ends of broken chromosomes 

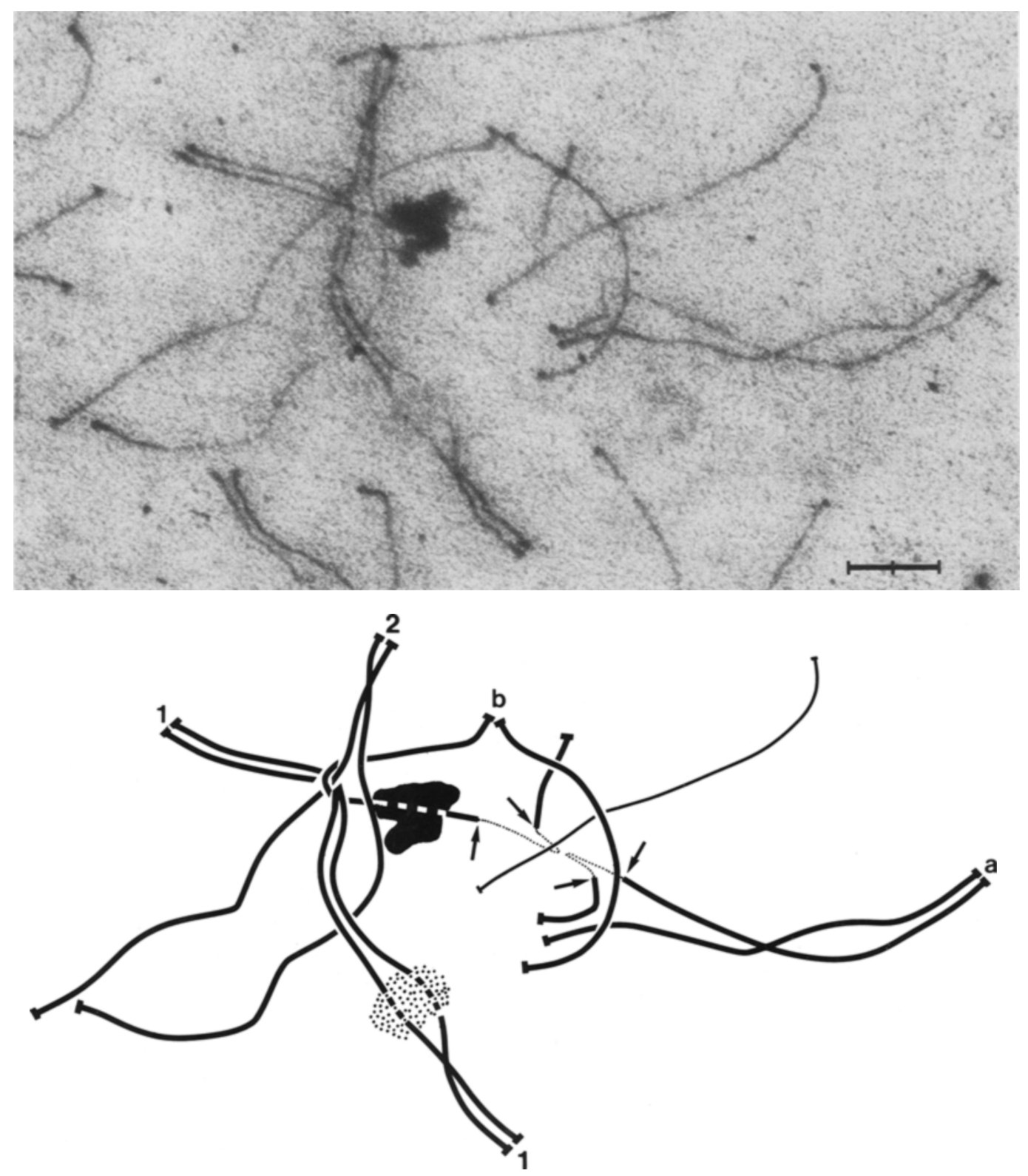

Figure 22. Free lateral component ends (arrows) of one homologue of each of bivalents a and $\mathbf{b}$. Most likely the free ends are due to resolution of a multiple interlocking involving bivalents $1,2, a$ and $b$. The drawing indicates the presumptive configuration. $(\mathrm{Bar}=2.0 \mu \mathrm{m})$

removed from the position in the nucleus where a break originally occurred. Mechanical breakage of LCs caused by the spreading procedure appeared to be rare as demonstrated by the preservation of LC continuity even in excessive- ly stretched LCs (see for example Figure 8). Except for the high frequency in nuclei $0-10 \%$ paired, the frequency of free LC ends and of resolving ILs (configurations of type 7, Figure 16 ) is fairly constant during zygotene (curves 2 


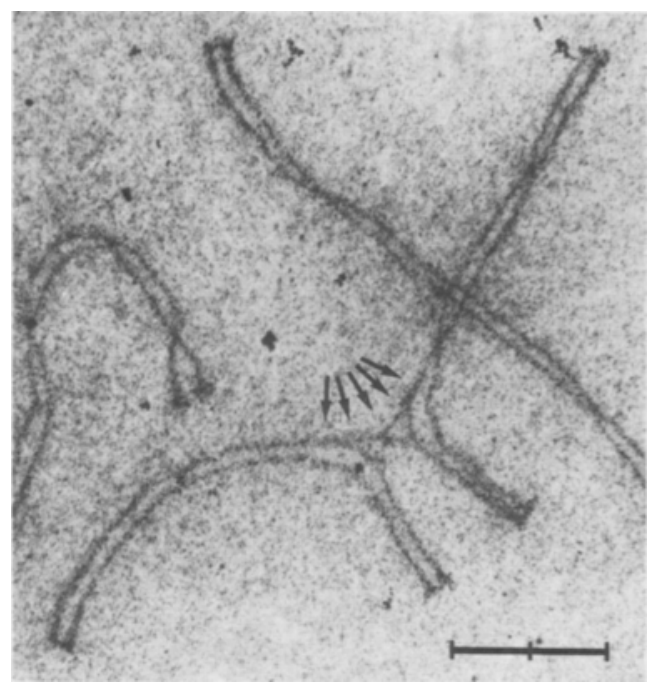

Figure 23. Presumptive intermediate in the resolution of an interlocking at pachytene stage 1 . The interlocked lateral components are longitudinally split at the site of the interlocking. Continuity of one "half"- $\mathrm{LC}$ has been reestablished in both bivalents while the other "half"LCs are still interlocked (see text). $($ Bar $=2.0 \mu \mathrm{m})$

and 3, Figure 20). It should be added though that the identification of resolving ILs in most instances is difficult in the spread complements and that the frequencies are subject to considerable uncertainty.

It appears from configurations as those shown in Figures 17 and 18 that resolution of ILs occurs in two phases: The first step is the release/passage of the interlocked LC and the reestablishment of the $\mathbf{L C}$ continuity. This is followed by passage of the chromatin of the involved chromosomes. In Figure 18 the LCs of the presumptive interlocked chromosomes have passed each other and have been repaired while the two chromosomes still appear to be associated at the chromatin level. Another example is shown in Figure 23 in which the LCs are locally split, one half of each LC having passed each other while the second half-LCs are still interlocked. Configurations of this type are rare and may either represent an intermediate stage in the normal resolution process or reflect an anomalous situation.

Extensive splitting of LCs was only observed in a single zygotene nucleus in which apparently normal central regions of the SC have formed between the two LC segments of each homologue in some of the bivalents (Figure 24). A single zygotene nucleus of similar morphology was found by BOJKO (4) among gamma-irradiated Bombyx spermatocytes. It is not known whether the double LCs and the aberrant SC formation in these bivalents are the result of over-replication of the DNA in some chromosome segments during premeiotic S-phase or are due to reduced sister chromatid affinity in the affected regions followed by central region formation between the two half-LCs.

\subsection{Pachytene}

Only 3 presumptive ILs were detected in the 125 spread pachytene complements. As pachytene nuclei were primarily identified by the absence of unpaired LC segments it cannot be entirely excluded that additional ILs may be present at early pachytene (in nuclei erroneously assigned to late zygotene, see section 3.2). If the terminal LC modification of chromosomes 1 and 2 in the spread material can be considered a reliable indicator of progression into pachytene, the 40 pachytene 1 complements contained two possible ILs (Figure 25), the 67 pachytene 2 complements only one presumptive IL and the 18 pachytene 3 complements none at all.

The reconstruction analysis of Bombyx spermatocytes (28) in which the the identification of pachytene substages is based on additional nuclear parameters other than the completion of the SC, revealed 2 ILs in 16 nuclei at early pachytene and none in 17 mid-late pachytene nuclei in agreement with the present results. It is thus likely that detection and resolution of ILs are possible during the first part of pachytene and hence that very few ILs remain after pachytene.

A spread pachytene complement is shown in Figure 26. Each pair of homologues is held together by a continuous SC extending from telomere to telomere and entanglements in the form of ILs and intertwinings are all resolved. and repaired. 


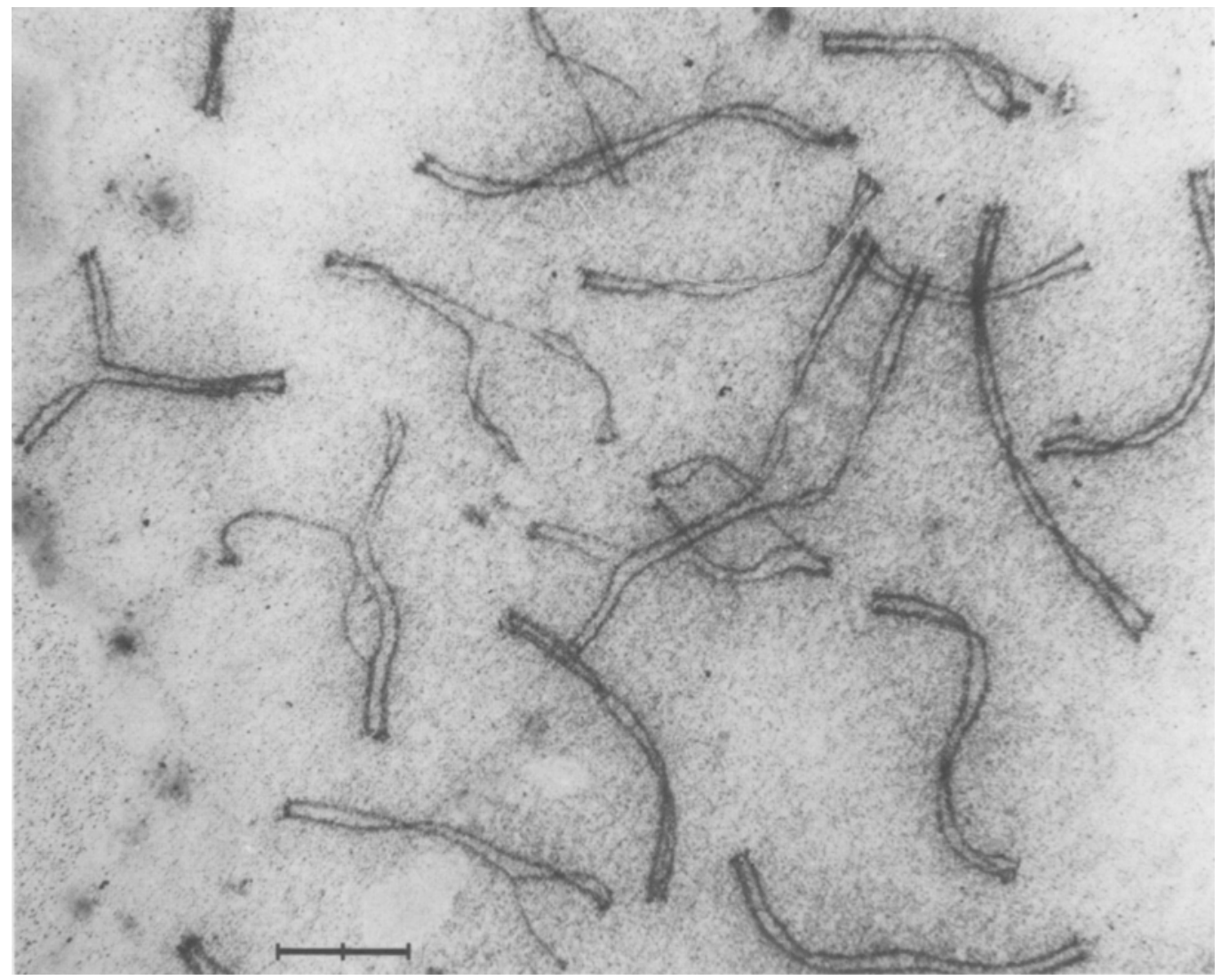

Figure 24. Partial and fully synapsed bivalents of an aberrant nucleus. Several stretches of lateral components are longitudinally split, often with an apparently normal central region between the two "half"-LCs. In some bivalents the splitting has affected about equal lengths of the two homologous LCs while in others splitting has occurred in only one homologue. $(\mathrm{Bar}=2.0 \mu \mathrm{m})$

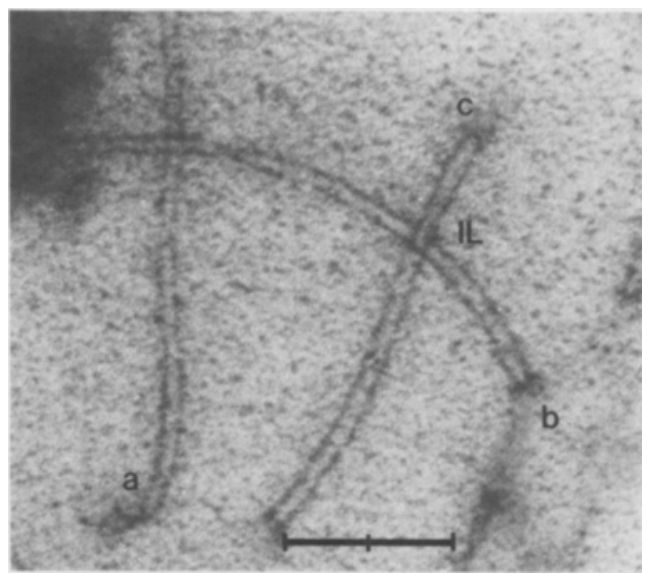

\section{DISCUSSION}

\subsection{The procedure}

As mentioned in section 3.2 and shown in Table I the number of analysable nuclei per group increases with the extent of SC formation. This reflects the difficulties in obtaining well preserved complements at early zygotene stages

\footnotetext{
Figure 25. Possible unresolved interlocking (IL) at pachytene stage 1 . Unlike the crossing of the synaptonemal complexes of bivalents a and $b$, one lateral component of bivalent $b$ appears to lie above and the other below the synaptonemal complex of the short bivalent $\mathrm{c} .(\mathrm{Bar}=2.0 \mu \mathrm{m})$
} 


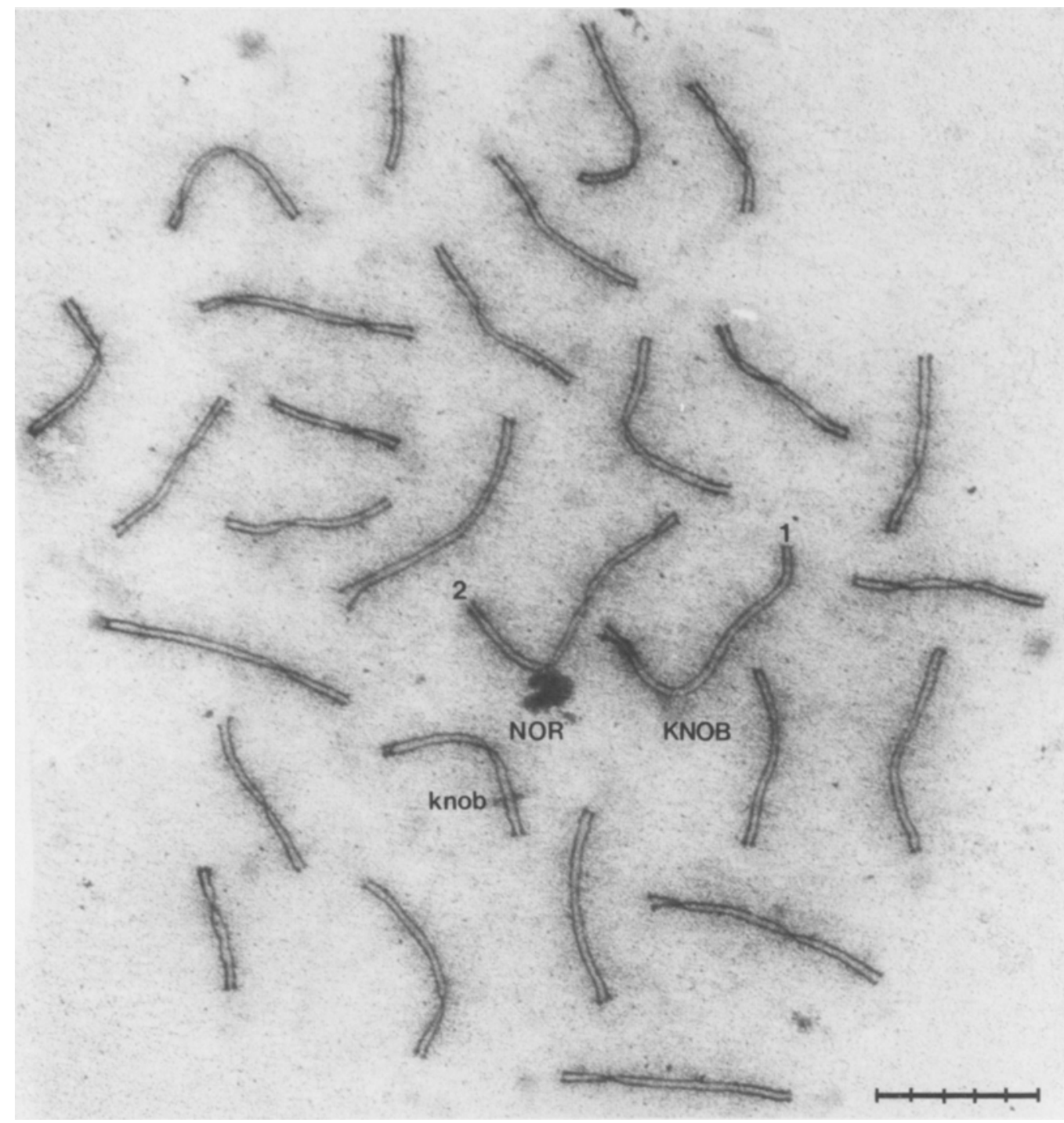

Figure 26. Fully paired chromosome complement at pachytene stage 1 . Bivalents 1,2 and the shorter knob-bearing bivalent are recognized by their morphological markers (KNOB, NOR and knob). Note the dense lateral component segments of bivalent 1 in the telomeric region near the knob. Compare with Figure $5 .(\operatorname{Bar}=5.0 \mu \mathrm{m})$

rather than a scarcity of these stages in the material. The same phenomenon was noted by HEYTING et al. (23) in their procedure for isolating SCs from rat spermatocytes. Following the separation of spermatocytes from other types of cells by gravity sedimentation, the spermatocytes were lysed in a buffered $1 \%$ Triton X-100, $0.5 \mathrm{~mm}$-EDTA solution. The swollen nuclei were digested by DNase II and the SCs collected on the interphase between Fluorinert FL-77 and $1.5 \mathrm{M}$-sucrose after centrifugation. Judged by the morphology of the isolated SCs it was apparent that mainly mid pachytene and older SC complements were preserved whereas only few SCs from younger spermatocytes contributed to the final isolate. Preliminary attempts to isolate SC 
from Bombyx spermatocytes have confirmed this observation. Treatment of isolated zygotene, pachytene and early diplotene Bombyx spermatocyte nuclei with $1 \%$ Triton $X-100$ effectively dissolves zygotene nuclei and render the SCs and LCs invisible in the light microscope while pachytene and older nuclei respond to the detergent treatment only by an increase in volume and transparency. The lower concentration of detergent used in the spreading procedure may thus account for the few acceptable spread early zygotene complements.

Methodological difficulties may also in part be responsible for the large range and standard deviation of the total LC length. The mean total LC length per nucleus for the entire zygotene period amounts to $438 \mu \mathrm{m}$ and is approximately the same as that determined for 8 sectioned late zygotene nuclei $(404 \mu \mathrm{m}, 28)$. The standard deviation is, however, about twice as large and the range four-fold larger (313-698 $\mu \mathrm{m}$ versus $354-444 \mu \mathrm{m}$ ) for the spread complements than for sectioned material.

\subsection{The interpretation of spread chromosome complements}

The interpretation of the topological relationship of the individual chromosomes of the spread complements is in most cases straight forward and confirms the observations and conclusions based on serially sectioned and reconstructed nuclei. The mean frequency of ILs (including multiple ILs and resolving ILs) in 8 reconstructed late zygotene nuclei (mean: $89 \%$ pairing, range: $75-98 \%$ ) amounted to $3.4(28)$. The corresponding value for 145 spread nuclei covering the same range of pairing (mean: $87 \%$, range: $70-99 \%$ ) is 1.6 . The frequencies of ILs determined by the two methods are of the same order of magnitude although the large standard deviation and range (Figures 11, 20 and 21) of the frequencies and the difference in number of nuclei analysed, do not warrant a statistical comparison. The higher frequency of ILs in the sectioned material indicates that some presumptive ILs not counted in the spread chromosome complements are true ILs.

\subsection{Synapsis and synaptonemal complex formation}

The analysis of early zygotene chromosomes from Bombyx spermatocytes revealed two distinctly different types of associations between $L C$ regions not yet paired with an SC. In the first type, association was observed between homologous chromosomes (i.e., chromosomes with LCs of the same length) at a short subterminal region holding the two homologous regions at a distance 2-3 times the width of the fully formied central region of the SC. The second type of association involves the dense attachment plaque of two of more LCs held together at a distance about equal to the width of the central region of the SC. This type of association appears to be indifferent to homology of the involved chromosomes.

The images observable after spreading indicate that SC formation often starts from associated attachment plaques of homologues already matched by subtelomeric associations at one or both ends. Bivalents with a $1-3 \mu \mathrm{m}$ SC segment at one or both ends prevail among partially paired homologues. This is consistent with telomeric initiation of SC formation and furthermore indicates a temporal delay/arrest of the progression of SC formation at the subterminal association sites. Interstitial SC formation not reaching the telomeres was extremely rare.

In the absence of ILs and intertwinings, SC formation progresses in a regular fashion once initiated near the telomeres and was only observed to involve homologous chromosomes in the present material. The few configurations resembling associations of four chromosomes (for example chromosomes 18 and 19 in Figure 3 ) could equally well be (and were) interpreted as interlocked bivalents.

Subtelomeric synaptic initiation was first described by MOENS (36) in Locusta spermatocytes. In this species two LCs (= axial cores) not yet combined by an SC were observed to be associated by traversing filaments spanning the $300 \mathrm{~nm}$ wide distance between subterminal segments of the LCs. Partial reconstructions of the region of telomeric attachment to the nuclear envelope at early zygotene identified subterminal SC initiation in many bivalents and in some cases revealed SC formation only in the 
region between the subterminal initiation sites and the telomeres. A recent reexamination of synapsis in Locusta and Schistocerca (32) based on spread chromosome complements $(10-80 \%$ pairing in Locusta and $10-69 \%$ pairing in Schistocerca) showed that SC initiation occurred predominantly but not entirely near the telomeres, confirming MOENS' original observations. Subterminal association preceding SC formation can also be inferred from published micrographs and reconstructions of early zygotene nuclei of the deer mouse, Peromyscus (19), the mouse (20) and Homo (44) in which pairs of homologues at early zygotene are associated subterminally at a distance exceeding the width of the completed central region of the SC.

Hence it seems probable that, at least in some organisms with relatively short chromosomes, the initial matching of homologous chromosomes occurs at a small number of unique recognition sites located subterminally or terminally in the chromosomes.

More complex and less amenable to a complete analysis are the spread or reconstructed early zygotene chromosome complements of the plant species investigated so far. Spread zygotene complements of Secale (16), Tradescantia $(21,22)$ and Triticum $(27,55)$ among others do, however, reveal chromosome associations in regions not yet combined by an SC resembling those described in the present analysis.

A more favorable situation exists in autotriploids. At a given site, SC formation normally occurs only between two of the three homologues. If the third LC retains its initial associations to one or both of its homologues, the sites are detectable during zygotene and early pachytene without being obscured by SC formation. Using this approach in Allium $(3 x=24)$, LOIDL and JONES (35) estimated the number of association sites to be between 20 and 30 per trivalent at early pachytene. The mean LC length of one genome is $1099 \mu \mathrm{m}$ which gives a mean distance between association sites of approximately $4.5 \mu \mathrm{m}$. The number of associations decreased during pachytene and by late pachytene none could be identified. Comparable mean distances between initiation sites for SC formation have been reported for Lilium (21,
26), Tradescantia (21), Secale $(1,16)$ and Triticum (27) the distances amounting to 6.5 $\mu \mathrm{m}, 9.1 \mu \mathrm{m}, 4.4-4.7 \mu \mathrm{m}$ and $9 \mu \mathrm{m}$, respectively, i.e., distances similar to those between the two subterminal sites of an average Bombyx bivalent.

Reconstruction of achiasmatic autotriploid Bombyx oocytes (43) revealed trivalents with one switch of pairing partner and trivalents in which one homologue was associated at one or both ends to a fully synapsed pair. This confirms the existence of two association sites per chromosome. The trivalents convert into a bivalent and a univalent during pachytene either by elimination of the primary associations or by turn-over of the SC in trivalents with a switch of pairing partner. The released univalents subsequently engage in various nonhomologous associations by nonspecific SC formation exclusively initiated at or near the telomeres of the chromosomes.

The temporal separation of the specific and nonspecific $\mathrm{SC}$ formation in triploid Bombyx is consistent with the existence of two principally different types of synaptic initiation preceding $\mathrm{SC}$ formation. It further shows that the specific initiation of SC formation is confined to zygotene while the additional nonspecific initiation takes place after zygotene. As the chromosomes available for the second round of SC formation at pachytene are all nonhomologous in a triploid, their pairing behaviour does not reveal whether $\mathrm{SC}$ formation at pachytene is entirely indifferent to homology.

The observation that the primary association can combine the three homologous chromosomes in a triploid (43) and all four in a tetraploid (45) finally suggests that the two sister chromatids of a chromosome each carry a copy of the recognition sequences.

In the grasshopper, Chloealtis, telomeric or subtelomeric specific initiation of synapsis is the rule (37). The occasional occurrence of interstitial SC segments does, however, show that interstitial initiation sites for synapsis exist but are usually not expressed. The existence of up to 7-9 interstitial sites for synaptic initiation per nucleus in Locusta $(2 n=22+X)$ and Schistocerca $(2 n$ $=22+X)$ was inferred from the presence of SC segments (32) and confirmed in Locusta by the 
occurrence of quadrivalents with up to two shifts of pairing partner in reconstructed autotetraploid spermatocyte nuclei (S.W. RASMUSSEN, K. CARBONE, L. CARBONE, unpublished observations).

In an attempt to explain apparently illegitimate chiasma formation between chromosomes 7 and 8 in spermatocytes of Chloealtis $(2 n=$ $16+\mathrm{X})$, MoENs (37) proposed that the specific association of homologues occurred prior to $\mathrm{SC}$ formation at small chromosome segments, socalled "synaptic units" and that the anomalous synapsis preceding chiasma formation between chromosomes 7 and 8 was due to the presence of homologous "synaptic units" on the heterologous chromosomes 7 and 8. Lateral displacement of homologous centromeres (separated $0-3 \mu \mathrm{m}$ ) was frequently observed in completely paired bivalents at pachytene in Chloealtis (37) implying that the subsequent SC formation extending from the initiation sites occurs in an undiscriminating fashion.

Additional analyses are required to establish whether association sites are located at constant positions in a given chromosome in different nuclei as appears to be the case for Bombyx chromosomes and perhaps also for the relatively short chromosomes of Sordaria (57), Locusta (37) and Homo (44). Although difficult to analyse systematically, it appears from the spread early zygotene Bombyx spermatocytes that homologous subterminal association sites are located equidistantly from the telomeres, whereas this is not the case for nonhomologous association sites (Figures 6 and 8). With the telomeres attached within the bouquet region, a chromosome specific distance between the telomere and the recognition site would confine the movements of homologous recognition sites to the same plane whereas nonhomologous sites would move in planes at different distances from the inner nuclear membrane. This would facilitate the initial matching of homologous recognition sites.

\subsection{Interlocking and resolution}

The analysis of synapsing chromosomes of Bombyx spermatocytes has confirmed that entanglement of chromosomes in the form of IL and intertwining occurs at high frequencies throughout the zygotene stage. It has further been shown that the entanglements are continuously resolved during zygotene by breakage and repair of the entangled chromosomes and bivalents. The frequency of chromosome ILs (curve 3 , Figure 21) reaches its maximum at $40-50 \%$ pairing and declines toward completion of synapsis whereas the frequency of bivalent ILs (curve 4, Figure 21) gradually increases during synapsis, reaches a maximum at $80-90 \%$ pairing and declines rapidly between 90 and 100\% pairing. Since the formation and resolution of ILs in a nucleus occur concomitantly, it is not possible to precisely estimate the frequency of chromosome breakage and reunion during zygotene in an average nucleus.

The combined maximum frequencies of ILs ( 2.2 types 3 and 4 at $40-50 \%$ pairing and 2.0 type 5 at $80-90 \%$ pairing) amount to 4.2 and represent a minimum estimate of the number of ILs an average nucleus will encounter during zygotene. The actual number of ILs is probably higher as indicated by the constant frequency of resolving IL and free LC ends (curves 2 and 3, Figure 20) throughout zygotene, implying that some ILs of both types may have been resolved before the maximum is reached.

Obviously, interlocking of chromosomes is a chance event and in individual nuclei, considerably higher and lower frequencies may be found. In a single nucleus at $53 \%$ pairing 34 chromosomes ( $61 \%$ of the complement) were affected by chromosome and bivalent ILs and chromosome breakage in presumptive interlocked regions at the time of fixation. In other nuclei at almost all substages of zygotene, ILs were entirely absent.

Although comparable quantitative data from other organisms are not available, it is evident from previous analyses of reconstructed or spread chromosome complements, that the frequent occurrence of ILs and breaks is not unique to Bombyx spermatocytes. ILs have been reported in nearly all organisms in which the zygotene stage has been studied (54). A fully reconstructed zygotene nucleus of Secale at $65 \%$ pairing contained 3 chromosome and 4 bivalent ILs affecting 12 of the 14 chromosomes (1). A similar number of ILs were found in spread complements of Secale (16). Spread zygotene 
complements of Triticum were reported to contain a minimum of 20 ILs most of which were bivalent ILs (27). ILs have previously been shown by reconstruction analyses to be common in spermatocytes of Homo (44) and Bombyx (28) and recently, 5 chromosome ILs, one bivalent IL and 2 free LC ends (probably signifying the resolution of an additional IL) were revealed in a reconstructed human oocyte nucleus at $67 \%$ pairing (3). These and additional studies $(4,9,11,14,20,24,27,55,58)$ are consistent with the present observations and confirm that chromosomes frequently interlock during synapsis and that such entanglements are normally resolved as synapsis progresses by release of the interlocked chromosomes through breakage and repair of the entangled chromosomes.

With the exception of Triticum (27), Chloealtis $(31,38)$ and possibly other organisms in which synapsis does not usually reach completion at pachytene, elimination of ILs prior to pachytene is the rule, implying that the resolution system normally detects and resolves ILs with high efficiency.

The observation that the resolution of an IL requires transient breakage of both sister chromatids implies a controlled process in which the passing chromosomes and their LC are held in register during the passage. This requirement inspired the proposal (28) that DNA topoisomerase II, which catalyses breakage, passage and rejoining of double stranded DNA (33), is involved in the passage of one chromosome across another chromosome or bivalent.

Recently, information on the properties of this enzyme has accumulated $(13,52)$ adding further evidence to the notion that topoisomerase II fulfils most of the requirements of an IL resolving system. The enzyme has been isolated and purified from several eukaryotic species $(13,34,52)$ including yeast $(17,18)$ and Drosophila (48). Several lines of evidence have furthermore established that topoisomerase II is identical to a major chromosome scaffold protein, $\mathrm{SC}$, isolated from HeLa cells (12). It has a molecular weight of about $170 \mathrm{kD}$ and comprises a remarkably large fraction (1-2\%) of the total chromosomal proteins corresponding to about 3 molecules per $70 \mathrm{~kb}$ of DNA. After extraction of the histones, from 60 to $80 \%$ of the topoisomerase II remains bound to the chromosome scaffold. Finally, it has been demonstrated that specific antibodies raised against $\mathrm{SCl}$ (= topoisomerase II) react intensely with the central axial region of the chromosome scaffold extending the length of isolated HeLa metaphase chromosomes. The reaction was observed whether or not the isolated chromosomes were depleted of histones (12).

Topoisomerase II has been shown to be indispensible for the regular progression of mitosis in yeast (25). When synchronized yeast cells carrying a temperature sensitive recessive mutation in the structural gene for topoisomerase II, top2, are shifted to the restrictive temperature the observed inviability coincides with the time of mitosis. HoLm et al. (25) further showed that the lethal effect of the top 2 mutation at the restrictive temperature is supressed if polymerization of tubulin is blocked by nocodazole and concluded: “... that it is the attempt by the cell to segregate the sister chromatids in the absence of topoisomerase II activity that leads to inviability". The experiments showed that the topoisomerase II activity in yeast is essential only at one point in the cell cycle. This does not rule out that topoisomerase II may have additional functions elsewhere in the cell, only that the failure of other possible functions is not fatal for the cell.

The suggestion that the inviability of mitotically dividing yeast cells in the absence of proper topoisomerase II function may be due to chromosome breakage or nondisjunction at anaphase (25) is supported by the observation of a 30-fold increased frequency of chromosome loss when yeast cells carrying the top $2-1$ mutation were maintained at a semipermissive temperature (HARTWELL, cited in 25).

DNA topoisomerase II activity has been identified throughout the zygotene stage and during the first part of the pachytene stage in lily microsporocytes (49) which, in the light of its role in the mitotic prophase, makes it likely that topoisomerase II is also involved in maintaining the integrity of individual chromosomes during synapsis at the meiotic prophase by enabling two interlocked or otherwise entangled chromosomes to pass each other.

It is evident from the spread early and mid 
zygotene complements of Bombyx that the detection of an IL does not require that SC formation progress until it reaches the foreign chromosome. Rather it appears that resolution is triggered if tension created by movements of the chromosomes in opposite directions during synapsis forces the two LC segments into close proximity. Many images of resolving ILs (Figures 18 and 19) suggest that passage of the two LC segments precedes the passage of the chromatin. It is at present not known whether the lagging chromatin association between previously interlocked chromosome segments signifies the actual passage of nonsister chromatids or whether the transient breakage occurs in the chromatin associated with the LCs.

In rare cases, ILs remain unresolved during zygotene. If crossing over and chiasma formation occur in appropriate segments of the interlocked bivalents, the IL may persist beyond pachytene $(6,27,56)$. A marked increase in the frequency of interlocked bivalents at metaphase I has been observed in Triturus (6) and Locusta (5) after exposure to increased temperature during zygotene. A similar increase was observed after injection of colchicine in pentaploid triticale (51) and as a result of altering the dose of the $P h l$ gene in hexaploid Triticum (56). In all cases the increase in the frequency of interlocked bivalents at metaphase I, although being significantly higher than in the controls, never approached the frequencies normally observed at zygotene in the wide range of organisms studied so far.

The frequency of IL at metaphase I is influenced by the number of IL actually formed at zygotene, by factors involved in the detection of ILs, by the proper function of the resolution system as well as by the number and distribution of crossovers at pachytene. External factors affecting any of these parameters may accordingly lead to an increased frequency of ILs at metaphase I. It has been shown that colchicine completely inhibits the rotatory chromosome movements normally accompanying synapsis in the male rat $(41,47)$. This would obviously affect chromosome pairing but would also reduce the tension on interlocked chromosomes and hence render the detection of an IL less efficient.
It is concluded that interlocking of chromosomes, rather than being avoided, regularly forms as homologous chromosomes synapse. Interlockings are subsequently detected and resolved by breakage and reunion of the involved chromosomes. The failure of detection or resolution of interlockings at zygotene in normal nuclei, whether occurring naturally or experimentally induced, can explain the occurrence of interlockings at metaphase I.

\section{ACKNOWLEDGEMENTS}

I wish to thank Professor DITER VON Wettstein and Drs. Preben B. Holm and DAVID J. SIMPSON for their critical review of the manuscript. The excellent technical assistance of BibI Stampe ANDERSEN. NinA RASMUSSEN and JEAN SAGE is gratefully acknowledged. The work was financially supported by grants BIO-E-417 DK(6) and BI6-168-DK from the Commission of the European Communities.

\section{REFERENCES}

1. AbiRACHEd-DARMENCY,M., D.ZICKLER \& Y.CAUDERON: Synaptonemal complex and recombination nodules in rye (Secale cereale). Chromosoma 88 , 299-306 (1983)

2. Bloom, S. E. \& C. Goodpasture: An improved technique for selective silver staining of nucleolar organizer regions in human chromosomes. Hum. Genet. 43, 199-206 (1976)

3. Bojko, M:: Human meiosis VIII. Chromosome pairing and formation of the synaptonemal complex in oocytes. Carlsberg Res. Commun. 48, 457-483 (1983)

4. Bолко, M.: Ultrastructural investigations of meiosis as a tool in assessing radiation damage in man. Thesis. University of Copenhagen, (1986)

5. Buss, M. E. \& S. A. Henderson: The induction of orientational instability and bivalent interlocking at meiosis. Chromosoma 35, 153-183 (1971)

6. Callan, H. G. \& S. M. Pearce: An experimental analysis of bivalent interlocking in spermatocytes of the newt Triturus vulgaris. J. Cell Sci. 37, 125-141 (1979)

7. Callan, H. G. \& P. E. Perry: Recombination in male and female meiocytes contrasted. Phil. Trans. R. Soc. Lond. B. 277, 227-233 (1977)

8. Comings D. E. \& A. D. RigGs: Molecular mechanisms of chromosome pairing, folding and func- 
tion. Nature 233, 48-50 (1971)

9. Dietrich, A. J. J. \& R. J. P. Mulder: A light- and electron microscopic analysis of meiotic prophase in female mice. Chromosoma 88, 377-385 (1983)

10. DRESSER, M. E.\& M. J. MOSES: Synaptonemal complex karyotyping in spermatocytes of the Chinese Hamster (Cricetulus griseus) IV. Light and electron microscopy of synapsis and nucleolar development by silver staining. Chromosoma 76, 1-22 (1980)

11. Freixa, L., M. Garcia, M. Ponsa, J. Navarro \& J. EGOzCUE: Sequential study of the synaptonemal complex in Syrian hamster (Mecocricetus auratus) and moụse (Mus musculus) oocytes by light and electron microscopy. Genetica 67, 87-97 (1985)

12. Gasser, S. M., T. Laroche, J. Falquet, E. Boy DE LA TOUR \& U. K. LAEMmLI: Metaphase chromosome structure. Involvement of topoisomerase II. J. Mol. Biol. 188, 613-629 (1986)

13. Gellert. M.: DNA topoisomerases. Ann. Rev. Biochem. 50, 879-910 (1981)

14. Gillies, C. B.: Electron microscopy of spread maize pachytene synaptonemal complexes. Chromosoma 83, 575-591 (1981)

15. Gillies. C. B.: Ultrastructural studies of the association of homologous and non-homologous parts of chromosomes in the mid-prophase of meiosis in Zea mays. Maydica 28, 265-287 (1983)

16. Gillies, C. B.: An electron microscopic study of synaptonemal complex formation at zygotene in rye. Chromosoma 92, 165-175 (1985)

17. Goto, T., P. LAIPIS \& J. C. WANG: The purification and characterization of DNA topoisomerase I and II of the yeast Saccharomyces cerevisiae. J. Biol. Chem. 259, 10422-10429 (1984)

18. Goto, T.\& J. C. WANG: Yeast DNA topoisomerase II. An ATP-dependent type II topoisomerase that catalyzes the catenation, decatenation unknotting, and relaxation of double-stranded DNA rings. J. Biol. Chem. 257, 5866-5872 (1982)

19. Greenbaum. I. F., D. W. Hale \& K. P. Fuxa: The mechanism of autosomal synapsis and the substaging of zygonema and pachynema from deer mouse spermatocytes. Chromosoma 93, 203-212 (1986)

20. Guitart, M., M. D. Coll, M. Ponsá \& J. Egozcue: Sequential study of synaptonemal complexes in mouse spermatocytes by light and electron microscopy. Genetica 67, 21-30 (1985)

21. Hasenkampr. C. A.: Synaptonemal complex formation in pollen mother cells of Tradescantia. Chromosoma 90, 275-284 (1984)

22. HaSenkampF, C. A. \& M. Y. Menzel: Synaptonemal complex karyotyping of Tradescantia zygotene nuclei. Amer. J. Bot. 72, 666-673 (1985)

23. Heyting, C., A.J.J. Dietrich, E. J. W. Redeker \& A.
C. G. VINK: Structure and composition of synaptonemal complexes, isolated from rat spermatocytes. Eur. J. Cell Biol. 36, 307-314 (1985)

24. Hobolth, P.: Chromosome pairing in allohexaploid wheat var. Chinese Spring. Transformation of multivalents into bivalents, a mechanism for exclusive bivalent formation. Carlsberg Res. Commun. 46, 129-173 (1981)

25. Holm, C., T. GoTo, J. C. WANG \& D. Botstein: DNA topoisomerase II is required at the time of mitosis in yeast. Cell 41, 553-563 (1985)

26. Holm, P. B.: Three dimensional reconstruction of chromosome pairing during the zygotene stage of meiosis in Lilium longiforum (Thunb. ). Carlsberg Res. Commun. 42, 103-151 (1977)

27. HoLM, P. B.: Chromosome pairing and chiasma formation in allohexaploid wheat, Triticum aestivum analyzed by spreading of meiotic nuclei. Carlsberg Res. Commun. 51, 239-294 (1986)

28. Holm, P. B. \& S. W. Rasmussen: Chromosome pairing, recombination nodules and chiasma formation in diploid Bombyx males. Carisberg Res. Commun. 45, 483-548 (1980)

29. Holm, P. B., S. W. RASMUSSEN, D. ZICKLER, B. C. LU \& J. SAGE: Chromosome pairing, recombination nodules and chiasma formation in the basidiomycete Coprinus cinereus. Carlsberg Res. Commun. 46, 305-346 (1981)

30. JenkiNs, G.: Chromosome pairing in Triticum aestivum cr. Chinese Spring. Carlsberg Res. Commun. 48, 255-283 (1983)

31. JoHN, B.: Myths and mechanisms of meiosis. Chromosoma 54, 295-325 (1976)

32. JoNeS, G. H. \& J. A. CROFT: Surface spreading of synaptonemal complexes in locusts II. Zygotene pairing behaviour. Chromosoma 93, 489-495 (1986)

33. LiU, L. F., C. C. LIU \& B. M. AlberTS: Type II DNA topoisomerases: Enzymes that can unknot a topologically knotted DNA molecule via a reversible double-strand break. Cell 19, 697-707 (1980)

34. LIU, L. F., T. C. ROWE, L. YANG, K. M. TEWEY \& G. L. CHEN: Cleavage of DNA by mammalian Topoisomerase II. J. Biol. Chem. 258, 15365-15370 (1983)

35. LOIDL, J. \& G. H. JONES: Synaptonemal complex spreading in Allium I. Triploid A. sphaerocephalon. Chromosoma 93, 420-428 (1986)

36. MoEns, P. B.: The fine structure of meiotic chromosome polarization and pairing in Locusta migratoria spermatocytes. Chromosoma 28, 1-25 (1969)

37. MoENs, P. B.: Research needs in meiosis, mechanisms of synapsis, and chiasma regulation. In: Aneuploidy, eds. V. L. Dellarco, P. E. Voytek \& A. Hollaender, 397-407, Plenum Publishing Corp. 
(1985)

38. MOENS, P. B. \& S. SHORT: Synaptonemal complexes of bivalents with localized chiasmata in Chloealtis conspersa (Orthoptera). Kew Chromosome Conference II, 99-106. George Allen \& Unwin (1983)

39. Moses, M. J. \& P. A. Poorman: Synaptonemal complex analysis of mouse chromosomal rearrangements II. Synaptic adjustment in a tandem duplication. Chromosoma 81, 519-535 (1981)

40. Moses, M. J., P. A. Poorman, T. H. Roderick \& M. T. DAvisson: Synaptonemal complex analysis of mouse chromosomal rearrangements IV. Synapsis and synaptic adjustment in two paracentric inversions. Chromosoma 84, 457-474 (1982)

41. Parvinen, M. \& K. - O. Söderström: Chromosome rotation and formation of synapsis. Nature 260 , 534-535 (1976)

42. Rasmussen, S. W.: The meiotic prophase in Bombyx mori females analyzed by three-dimensional reconstructions of synaptonemal complexes. Chromosoma 54, 245-293 (1976)

43. RASMUSSEN, S. W.: Chromosome pairing in triploid females of Bombyx mori analyzed by three dimensional reconstructions of synaptonemal complexes. Carlsberg Res. Commun. 42, 163-197 (1977)

44. RaSMUSSEN, S. W.\& P. B. Holm: Human meiosis II. Chromosome pairing and recombination nodules in human spermatocytes. Carlsberg Res. Commun. 43, 275-327 (1978)

45. Rasmussen, S. W. \& P. B. Holm: Chromosome pairing in autotetraploid Bombyx females. Mechanism for exclusive bivalent formation. Carlsberg Res. Commun. 44, 101-125 (1979)

46. SakaguChI, B.: Gametogenesis, fertilization and embryogenesis of the silkworm. In: The Silkworm: An Important Laboratory Tool, ed. Y. Tazima. pp. 5-30. Kodansha Tokyo (1978)

47. Salonen, K., J. Paranko \& M. Parvinen: A colcemid-sensitive mechanism involved in regulation of chromosome movements during meiotic pairing. Chromosoma 85, 611-618 (1982)

48. Shelton, E. R., N. Osheroff \& D. L. BrutlaG:
DNA topoisomerase II from Drosophila melanogaster. Purification and physical characterization. J. Biol. Chem. 258, 9530-9535 (1983)

49. STERn, H. \& Y. HotTa: Meiotic aspects of chromosome organization. Stadler Symposia 15, 25-41 (1983)

50. TEASE, C. \& G. H. JONES: Analysis of exchanges in differentialy stained meiotic chromosomes of Locusta migratoria after BrdU-substitution and FPG staining I. Crossover exchanges in monochiasmata bivalents. Chromosoma 69, 163-178 (1978)

51. Thomas, J. B. \& P. J. Kaltsikes: The effect of colchicine on chromosome pairing. Can. J. Genet. Cytol. 19, 231-249 (1977)

52. WANG, J. C.: DNA topoisomerases. Ann. Rev. Biochem. 54, 665-697 (1985)

53. Werrh, A. \& W. TRaUt: Synaptic adjustment, nonhomologous pairing, and nonpairing of homologous segments in sex chromosome mutants of Ephestia kuehniella (Insecta, Lepidoptera). Chromosoma 94, 125-131 (1986)

54. WetTSTEIN, D. von, S. W. Rasmussen \& P. B. Holm: The synaptonemal complex in genetic segregation. Ann. Rev. Genet. 18, 331-413 (1984)

55. WischmanN, B.: Chromosome pairing and chiasma formation in wheat plants triisosomic for the long arm of chromosome 5B. Carlsberg Res. Commun. 51, 1-25 (1986)

56. YacoBi, Y.Z., T. Mello-Sampayo \& M. Feldman: Genetic induction of bivalent interlocking in common wheat. Chromosoma 87, 165-175 (1982)

57. ZICKLER, D.: Development of the synaptonemal complex and the "recombination nodules" during the meiotic prophase of the seven bivalents of the fungus Sordaria macrospora Auersw. Chromosoma 61, 289-316 (1977)

58. ZiCKLeR, D. \& J. SAGE: Synaptonemal complexes with modified lateral elements in Sordaria humana: Development of and relationship to the "recombination nodules". Chromosoma 84, 305$318(1981)$ 